TRANSACTIONS OF THE

AMERICAN MATHEMATICAL SOCIETY

Volume 357, Number 3, Pages 1125-1159

S 0002-9947(04)03684-0

Article electronically published on October 7, 2004

\title{
WEAKLY COMPACT APPROXIMATION IN BANACH SPACES
}

\author{
EDWARD ODELL AND HANS-OLAV TYLLI
}

\begin{abstract}
The Banach space $E$ has the weakly compact approximation property (W.A.P. for short) if there is a constant $C<\infty$ so that for any weakly compact set $D \subset E$ and $\varepsilon>0$ there is a weakly compact operator $V: E \rightarrow E$ satisfying $\sup _{x \in D}\|x-V x\|<\varepsilon$ and $\|V\| \leq C$. We give several examples of Banach spaces both with and without this approximation property. Our main results demonstrate that the James-type spaces from a general class of quasi-reflexive spaces (which contains the classical James' space $J$ ) have the W.A.P, but that James' tree space JT fails to have the W.A.P. It is also shown that the dual $J^{*}$ has the W.A.P. It follows that the Banach algebras $W(J)$ and $W\left(J^{*}\right)$, consisting of the weakly compact operators, have bounded left approximate identities. Among the other results we obtain a concrete Banach space $Y$ so that $Y$ fails to have the W.A.P., but $Y$ has this approximation property without the uniform bound $C$.
\end{abstract}

\section{INTRODUCTION}

A Banach space $E$ is said to have the weakly compact approximation property (abbreviated W.A.P.) if there is a constant $C<\infty$ such that for any weakly compact set $D \subset E$ and $\varepsilon>0$ there is a weakly compact operator $V: E \rightarrow E$ satisfying

$$
\sup _{x \in D}\|x-V x\|<\varepsilon \quad \text { and } \quad\|V\| \leq C .
$$

This (bounded) weakly compact approximation property was introduced by Astala and Tylli $[\mathrm{AT}]$. The applications mentioned below were the principal motivation for this in $\mathrm{AT}$, but the W.A.P. is a natural notion worthy of study in its own right. Clearly any reflexive Banach space has the W.A.P., but this property is quite rare for non-reflexive spaces. For instance, if $E$ is a $\mathcal{L}^{1}$ - or $\mathcal{L}^{\infty}$-space, then $E$ has the W.A.P. if and only if $E$ has the Schur property; see [AT, Cor. 3]. We note that a different notion is obtained by considering the uniform approximation of the identity operator on compact sets by weakly compact operators (see e.g. Reinov [R], Grønbæk and Willis [GW], and Lima, Nygaard and Oja [LNO for this).

The weakly compact approximation property defined by (1.1) has some unexpected applications. The key fact [AT, Thm. 1] here is that the Banach space $F$ has the W.A.P. if and only if the measure of weak non-compactness

$$
\omega(S)=\inf \left\{\varepsilon>0: S B_{E} \subset D+\varepsilon B_{F}, D \subset F \text { weakly compact }\right\}
$$

Received by the editors September 25, 2003.

2000 Mathematics Subject Classification. Primary 46B28; Secondary 46B25, 46B45.

The first author's research was supported by the NSF.

The second author's research was supported by the Academy of Finland Project \# 53893. 
and the weak essential norm

$$
S \mapsto\|S\|_{w} \equiv \operatorname{dist}(S, W(E, F))
$$

are uniformly comparable in the space $L(E, F)$ of bounded linear operators $E \rightarrow F$ for all Banach spaces $E$. Here $B_{E}=\{x \in E:\|x\| \leq 1\}$ and $W(E, F)$ stands for the weakly compact operators $E \rightarrow F$. The fact that $c_{0}$ fails to have the W.A.P. was then applied in [AT, Thm. 4 and Cor. 5] to show that $\omega(S)$ is in general neither uniformly comparable to $\omega\left(S^{*}\right)$ nor to $\omega(J S)$ for arbitrary linear into isometries $J$. Subsequently, weakly compact approximation properties were exploited in [T2 to obtain examples of Banach spaces $E$ and $F$, where $\|S\|_{w}$ is not uniformly comparable to $\left\|S^{*}\right\|_{w}$. Further applications of the W.A.P. arise from the fact that the Banach algebra $W(E)$ has a bounded left approximate identity whenever $E$ has the W.A.P.

This paper contains several results and examples about the weakly compact approximation property for Banach spaces. A first natural question is in which sense "almost reflexive" Banach spaces still possess the W.A.P. A principal aim is to discuss the W.A.P. for the class of quasi-reflexive Banach spaces $E$, where $\operatorname{dim}\left(E^{* *} / E\right)<\infty$. In sections 2 and 3 we show that the classical James space $J$ and its dual $J^{*}$ have the W.A.P. These results imply that $W(J)$ and $W\left(J^{*}\right)$ have bounded left approximate identities. In this direction Loy and Willis [LW] established that $W(J)$ has a bounded right approximate identity. In section 4 we extend the results of section 2 by proving that the quasi-reflexive James-like spaces constructed by Bellenot, Haydon and Odell in $[\mathrm{BHO}$ have the W.A.P. These positive results are further highlighted by the recent discovery of Argyros and Tolias (see [ArT, Prop. 14.10]) that there exist quasi-reflexive hereditarily indecomposable Banach spaces $E$ that do not have the W.A.P.

In section 5 we present a permanence property for weakly compact approximation properties, which implies among other things that certain vector-valued sequence spaces, including $\ell^{1}\left(\ell^{p}\right)$ and $\ell^{p}\left(\ell^{1}\right)$ for $1<p<\infty$, have the W.A.P.

Section 6 contains a number of additional examples of spaces failing the W.A.P. For instance, we show that James' tree space JT does not have the W.A.P. We also establish that the W.A.P. differs from the corresponding "unbounded" W.A.P. (where the uniform bound $\|V\| \leq C$ is removed from (1.1)). Moreover, we obtain a concrete Banach space $Y$ so that the quotient $Y^{* *} / Y$ is isometric to $\ell^{2}$, but $Y$ does not have the W.A.P. (This example yields a simpler negative answer to a question from [AT] than the quasi-reflexive spaces constructed in [ArT].) Another natural problem is whether $E$ always has the W.A.P. if $E$ is $\ell^{1}$-saturated and $E$ has a Schauder basis. Indeed this is false, since as we show the Lorentz sequence spaces $d(w, 1)$, as well as the Azimi-Hagler spaces from $[\mathbf{A H}]$, do not have the W.A.P.

The basic terminology and notation related to Banach spaces will follow [LT.

\section{The James space $J$ has the W.A.P.}

The most well-known (and first discovered) quasi-reflexive Banach space $J$ was introduced by James [J1]. The fact that $J$ has the W.A.P. follows from the more general results of section 4 . However, that argument is more complicated and many of the ideas we use there, and in section 3, are well illustrated by first presenting them for $J$. 
Recall that a real-valued sequence $x=\left(x_{j}\right) \in J$ if $\lim _{j \rightarrow \infty} x_{j}=0$ and the square variation norm

$$
\|x\|^{2}=\sup \sum_{j=1}^{n}\left|x_{p_{j+1}}-x_{p_{j}}\right|^{2}<\infty,
$$

where the supremum is taken over all indices $1 \leq p_{1}<p_{2}<\ldots<p_{n}<p_{n+1}$ and $n \in \mathbf{N}$. The monograph $[\mathrm{FG}$ is a convenient source of results (as well as further references) about $J$. Recall that the coordinate basis $\left(e_{n}\right)$ is a shrinking Schauder basis for $J$, so that $J^{* *}$ can be identified with the set of scalar sequences $x=\left(x_{j}\right)$ for which $\sup _{n}\left\|\sum_{j=1}^{n} a_{j} e_{j}\right\|<\infty$. Moreover, $J^{* *}=\{x+\lambda \mathbf{1}: x \in J, \lambda \in \mathbf{R}\}$, where $\mathbf{1}=(1,1,1, \ldots)$.

The question whether (1.1) is satisfied for any weakly compact subset $D \subset J$ can be viewed as a concrete approximation problem for $J$ that may have independent interest. The set $\left\{e_{n}: n \in \mathbf{N}\right\} \cup\{0\}$ of $J$ is already a non-trivial test for (1.1), since the sequence $\left(e_{n}\right)$ is weakly null in $J$. It turns out that (somewhat surprisingly) the desired approximating operators $V \in W(J)$ are perturbations of the identity operator by certain double averaging functionals over consecutive blocks.

We first state a well-known general auxiliary result. It is convenient to put $[n, m)=\{n, \ldots, m-1\}$ if $m, n \in \mathbf{N}$ and $m>n$.

Lemma 2.1. Suppose that $E$ is a Banach space with a normalized Schauder basis $\left(e_{n}\right)$, and let $D \subset E$ be an arbitrary weakly compact subset. Then for any $\delta>0$ and $n \in \mathbf{N}$ there is $m>n$ such that for any $x=\sum_{j=1}^{\infty} a_{j} e_{j} \in D$ there is an index $j=j(x) \in[n, m)$ satisfying $\left|a_{j}\right|<\delta$.

Proof. Suppose to the contrary that there is $\delta>0$ and $n \in \mathbf{N}$ so that for any $m>n$ there is an element $x_{m}=\sum_{j=1}^{\infty} a_{j}^{(m)} e_{j} \in D$ satisfying $\left|a_{j}^{(m)}\right| \geq \delta$ for all $j \in[n, m)$. By the weak compactness of $D$ we may assume that $x_{m} \stackrel{w}{\longrightarrow} x=\sum_{j=1}^{\infty} a_{j} e_{j} \in D$ as $m \rightarrow \infty$. Since $a_{j}=\lim _{m \rightarrow \infty} a_{j}^{(m)}$ for $j \in \mathbf{N}$, we arrive at the contradiction that $\left|a_{j}\right| \geq \delta$ for each $j \geq n$.

Theorem 2.2. James' space J has the W.A.P.

Proof. The argument will be split into several steps. Let $D \subset J$ be a fixed weakly compact subset and $\varepsilon>0$. By homogeneity there is no loss of generality to assume that $D \subset B_{J}$.

Step 1. We start by fixing some notation. Given natural numbers $1 \leq n_{1}<\ldots<$ $n_{k+1}$ we introduce the related averaging functionals $A_{\left[n_{j}, n_{j+1}\right)}$ and $A_{\left(n_{1}, \ldots, n_{k+1}\right)}$ on $J$ by

$$
A_{\left[n_{j}, n_{j+1}\right)}(x)=\frac{1}{n_{j+1}-n_{j}} \sum_{s=n_{j}}^{n_{j+1}-1} a_{s}, \quad A_{\left(n_{1}, \ldots, n_{k+1}\right)}(x)=\frac{1}{k} \sum_{j=1}^{k} A_{\left[n_{j}, n_{j+1}\right)}(x),
$$

for $x=\sum_{i=1}^{\infty} a_{i} e_{i} \in J$ and $j=1, \ldots, k$. Note that $\left\|A_{\left[n_{j}, n_{j+1}\right)}\right\| \leq 1$ for each $j$, since

$$
\left|A_{\left[n_{j}, n_{j+1}\right)}(x)\right|=\frac{1}{n_{j+1}-n_{j}}\left|\left\langle\sum_{s=n_{j}}^{n_{j+1}-1} e_{s}^{*}, x\right\rangle\right| \leq\|x\| .
$$

Here $\left(e_{s}^{*}\right) \subset J^{*}$ stands for the sequence of biorthogonal functionals to $\left(e_{s}\right)$; clearly $\left\|e_{s}^{*}\right\|=1$ for $s \in \mathbf{N}$. We next show that certain double averages $A_{\left(n_{1}, \ldots, n_{k+1}\right)}$ are uniformly small on the weakly compact set $D$, provided $k$ is large enough. 
Claim 1. Let $\delta>0$ and $n=n_{1} \in \mathbf{N}$ be arbitrary. Then there is $k \in \mathbf{N}$ and natural numbers $n_{1}<\ldots<n_{k+1}$ so that

$$
\left|A_{\left(n_{1}, \ldots, n_{k+1}\right)}(x)\right|<3 \delta \quad \text { for all } x \in D \text {. }
$$

Proof of Claim 1. Use Lemma 2.1 repeatedly to choose a sequence $n=n_{1}<$ $n_{2}<\ldots$ in $\mathbf{N}$, such that for any $j \in \mathbf{N}$ and $x=\sum_{i=1}^{\infty} a_{i} e_{i} \in D$, there is an index $i=i(x) \in\left[n_{j}, n_{j+1}\right)$ satisfying $\left|a_{i}\right|<\delta$. Let $k \in \mathbf{N}$ be given. For each fixed $x=\sum_{i=1}^{\infty} a_{i} e_{i} \in D$ put

$$
I=\left\{j \leq k: \text { there is } i \in\left[n_{j}, n_{j+1}\right) \text { with }\left|a_{i}\right| \geq 2 \delta\right\}
$$

(note that $I$ depends on $x, k$ and $\delta$ ). In order to choose $k$ suppose that $I=$ $\left\{j_{1}, \ldots, j_{r}\right\}$ and pick $p_{s}, q_{s} \in\left[n_{j_{s}}, n_{j_{s}+1}\right)$ such that $\left|a_{p_{s}}\right|<\delta$ and $\left|a_{q_{s}}\right| \geq 2 \delta$ for $s=1, \ldots, r$. The square variation norm (2.1) satisfies

$$
\|x\| \geq\left(\sum_{s=1}^{r}\left|a_{q_{s}}-a_{p_{s}}\right|^{2}\right)^{1 / 2} \geq|I|^{1 / 2} \delta .
$$

We deduce that the cardinality $|I| \leq \frac{1}{\delta^{2}}$, because $D \subset B_{J}$ by assumption. Note further that $\left|A_{\left[n_{j}, n_{j+1}\right)}(x)\right|<2 \delta$ whenever $j \notin I$, since $\left|a_{s}\right|<2 \delta$ for all $s \in\left[n_{j}, n_{j+1}\right)$ in this event. By putting these estimates together we get that

$$
\left|A_{\left(n_{1}, \ldots, n_{k+1}\right)}(x)\right| \leq \frac{1}{k}(|I|+(k-|I|) 2 \delta) \leq \frac{1}{k \delta^{2}}+2 \delta<3 \delta
$$

once we pick $k>\delta^{-3}$. The completes the argument for Claim 1 .

Step 2. Fix a decreasing null-sequence $\left(\varepsilon_{j}\right)$ such that $\sum_{j=1}^{\infty} \varepsilon_{j}<\varepsilon / \sqrt{2}$. By successive applications of Claim 1 we find a sequence of consecutive subdivisions $1=n_{p_{1}}<n_{p_{1}+1}<\ldots<n_{p_{2}}<n_{p_{2}+1}<\ldots<n_{p_{3}}<n_{p_{3}+1}<\ldots$ of $\mathbf{N}$ such that

$$
\left|A_{j}(x)\right|<\varepsilon_{j} \quad \text { for all } x \in D \text { and } j \in \mathbf{N} \text {, }
$$

where we set $A_{j}=A_{\left(n_{p_{j}}, n_{p_{j}+1}, \ldots, n_{p_{j+1}}\right)}$ for $j \in \mathbf{N}$. Let $I_{j}=\left[n_{p_{j}}, n_{p_{j+1}}\right)$ for $j \in \mathbf{N}$, which is the "support" (with respect to the coordinate basis $\left(e_{s}\right)$ ) of the functional $A_{j}$ on $J$. Put $g_{j}=\sum_{i \in I_{j}} e_{i}$, so that $\left\|g_{j}\right\| \leq \sqrt{2}$ for $j \in \mathbf{N}$. Define the linear map $V$ on $J$ by

$$
V x=x-\sum_{j=1}^{\infty} A_{j}(x) g_{j}, \quad x \in J .
$$

We verify in three separate steps that $V$ provides a uniformly bounded weakly compact approximating operator for the given weakly compact set $D \subset B_{J}$ as required by (1.1).

Claim 2. $\|x-V x\|<\varepsilon$ for all $x \in D$.

Proof of Claim 2. It follows from (2.3) and the choice of $\left(\varepsilon_{j}\right)$ that

$$
\|x-V x\|=\left\|\sum_{j=1}^{\infty} A_{j}(x) g_{j}\right\| \leq \sum_{j=1}^{\infty}\left|A_{j}(x)\right| \cdot\left\|g_{j}\right\|<\sqrt{2} \cdot \sum_{j=1}^{\infty} \varepsilon_{j}<\varepsilon .
$$

Claim 3. $\|V\| \leq 3$ (independently of the subdivisions).

Proof of Claim 3. Put $\tilde{V} x=\sum_{j=1}^{\infty} A_{j}(x) g_{j}$ for $x \in J$. It suffices to verify that $\|\tilde{V}\| \leq 2$. 
It is convenient to denote $y=\sum_{j=1}^{\infty} y(j) e_{j} \in J$ in the argument. Assume that $x \in J$ is finitely supported, and suppose that $q_{1}<\ldots<q_{n+1}$ is a sequence of coordinates that realizes the square variation norm (2.1) of $\tilde{V} x$. We first split

$$
\begin{gathered}
\|\tilde{V} x\|=\left(\sum_{i=1}^{n}\left|\tilde{V} x\left(q_{i+1}\right)-\tilde{V} x\left(q_{i}\right)\right|^{2}\right)^{1 / 2} \\
\leq\left(\sum_{i \in A}\left|\tilde{V} x\left(q_{i+1}\right)-\tilde{V} x\left(q_{i}\right)\right|^{2}\right)^{1 / 2}+\left(\sum_{i \in B}\left|\tilde{V} x\left(q_{i+1}\right)-\tilde{V} x\left(q_{i}\right)\right|^{2}\right)^{1 / 2},
\end{gathered}
$$

where $i \in A$ if both $q_{i}, q_{i+1} \in I_{j}$ for some $j$, while $i \in B$ if $q_{i}$ and $q_{i+1}$ belong to different intervals. Note that if $i \in A$ and $q_{i}, q_{i+1} \in I_{j}$, then $\left|\tilde{V} x\left(q_{i+1}\right)-\tilde{V} x\left(q_{i}\right)\right|=$ $\left|A_{j}(x)-A_{j}(x)\right|=0$ by definition, so that the term $\left(\sum_{i \in A}\left|\tilde{V} x\left(q_{i+1}\right)-\tilde{V} x\left(q_{i}\right)\right|^{2}\right)^{1 / 2}$ actually vanishes. To estimate the second term in (2.4) we split

$$
\begin{aligned}
\left(\sum_{i \in B}\left|\tilde{V} x\left(q_{i+1}\right)-\tilde{V} x\left(q_{i}\right)\right|^{2}\right)^{1 / 2} \leq & \left(\sum_{i \in B_{1}}\left|\tilde{V} x\left(q_{i+1}\right)-\tilde{V} x\left(q_{i}\right)\right|^{2}\right)^{1 / 2} \\
& +\left(\sum_{i \in B_{2}}\left|\tilde{V} x\left(q_{i+1}\right)-\tilde{V} x\left(q_{i}\right)\right|^{2}\right)^{1 / 2} .
\end{aligned}
$$

In (2.5) the set $B_{1}$ contains every $2 k+1$-th term of $B$, and $B_{2}$ the remaining ones. Consider a single term $\left|\tilde{V} x\left(q_{i+1}\right)-\tilde{V} x\left(q_{i}\right)\right|$ for some $i \in B_{1}$, and suppose that $q_{i} \in I_{j}$ and $q_{i+1} \in I_{k}$ (where $j<k$ ). In this case

$$
\left|\tilde{V} x\left(q_{i}\right)-\tilde{V} x\left(q_{i+1}\right)\right|=\left|A_{j}(x)-A_{k}(x)\right| .
$$

By definition the double average $A_{j}(x)$ is a convex combination of $\left\{x(s): s \in I_{j}\right\}$ (and analogously for $A_{k}(x)$ ). Consequently there are indices $r_{i} \in I_{j}$ and $r_{i+1} \in I_{k}$ so that $\left|A_{j}(x)-A_{k}(x)\right| \leq\left|x\left(r_{i}\right)-x\left(r_{i+1}\right)\right|$. Since $B_{1}$ contains every second index from $B$, it is easy to check that the corresponding sequence $\left(r_{i}\right)$ is increasing, so we obtain that $\left(\sum_{i \in B_{1}}\left|A_{j}(x)-A_{k}(x)\right|^{2}\right)^{1 / 2} \leq\|x\|$. By arguing in a similar manner for the sum over the "even" indices $i \in B_{2}$, we get from (2.5) that

$$
\|\tilde{V} x\|=\left(\sum_{i \in B}\left|\tilde{V} x\left(q_{i+1}\right)-\tilde{V} x\left(q_{i}\right)\right|^{2}\right)^{1 / 2} \leq 2\|x\| .
$$

Hence we get by approximating that $\|\tilde{V} x\| \leq 2\|x\|$ for all $x \in J$. This establishes Claim 3.

Claim 4. $\quad V \in W(J)$.

Proof of Claim 4. Recall that $V \in W(J)$ if and only if $V^{* *}(\mathbf{1}) \in J$, where $\mathbf{1}=(1,1, \ldots) \in J^{* *} \backslash J$. Put $f_{m}=\sum_{j=1}^{m} e_{j}$ for $m \in \mathbf{N}$. Note that $f_{m_{k}} \stackrel{w^{*}}{\longrightarrow} \mathbf{1}$ and $V f_{m_{k}} \stackrel{w^{*}}{\longrightarrow} V^{* *} \mathbf{1}$ in $J^{* *}$ as $k \rightarrow \infty$ for each subsequence $\left(f_{m_{k}}\right)$ of $\left(f_{m}\right)$. Consider $s_{k}=\sum_{j=1}^{k} g_{j}$ for $k \in \mathbf{N}$, which determines a subsequence of $\left(f_{m}\right)$. Here

$$
V g_{j}=g_{j}-\sum_{k=1}^{\infty} A_{k}\left(g_{j}\right) g_{k}=0, \quad j \in \mathbf{N}
$$

since the averages $A_{k}\left(g_{j}\right)=\delta_{j, k}$ for $j, k \in \mathbf{N}$. It follows that $V s_{k}=0$ for $k \in \mathbf{N}$, so that $V^{* *} \mathbf{1}=0$. Thus $V \in W(J)$. This completes the proof of Theorem 2.2.

Remarks 2.3. (i) Simpler uniformly bounded approximating operators $V \in W(J)$ are available for the particular weakly compact set $D=\left\{e_{n}: n \in \mathbf{N}\right\} \cup\{0\} \subset J$. 
Indeed, define $V_{k} \in W(J)$ for $k \in \mathbf{N}$ by

$$
V_{k} x=x-\sum_{j=1}^{\infty} A_{[k j, k(j+1))}(x) h_{j}, \quad \text { where } h_{j}=\sum_{s=k j}^{k(j+1)-1} e_{s}, j \in \mathbf{N} .
$$

Then $V_{k}$ satisfies (1.1) for $D$ and $\varepsilon>0$ once $\frac{\sqrt{2}}{k}<\varepsilon$, since $\frac{1}{|I|}\left|\sum_{s \in I} x(s)\right| \leq \frac{1}{|I|}$ for intervals $I \subset \mathbf{N}$ and $x=\sum_{s=1}^{\infty} x(s) e_{s} \in D$. The uniform bound for $\left\|V_{k}\right\|$ and the weak compactness of $V_{k}$ are easy modifications of Claims 3 and 4 above.

Let $S_{k} \in L(J)$ be the forward $k$-shift on $J$ for $k=0,1,2, \ldots$. The reader may also wish to check that $V_{m}=I-\frac{1}{m+1} \sum_{k=0}^{m} S_{k} \in W(J)$ for $m \in \mathbf{N}$, and that $V_{m}$ satisfies (1.1) for $D$ and $\varepsilon>0$ once $m$ is large enough.

(ii) The argument of section 4 yields more complicated weakly compact approximating operators when applied to $J$ (e.g. the double averaging functionals are replaced by less explicit convex combinations, and there is a "shift"-like perturbation of the identity).

The following vector-valued analogue of James' space $J$ has been studied in several contexts; see e.g. $[\mathrm{PQ}]$ and $[\mathrm{P}]$. Let $E$ be a Banach space and $1<p<\infty$. The sequence $x=\left(x_{j}\right) \subset \bar{E}$ belongs to $J_{p}(E)$ if $\lim _{j \rightarrow \infty} x_{j}=0$ and the $p$-variation norm

$$
\|x\|^{p}=\sup _{n ; p_{1}<\ldots<p_{n+1}} \sum_{j=1}^{n}\left\|x_{p_{j+1}}-x_{p_{j}}\right\|^{p}<\infty .
$$

Here $J_{p}(E)^{* *} / J_{p}(E) \approx E$ for reflexive spaces $E$; see e.g. [W, Cor. 2]. Straightforward modifications of the argument in Theorem 2.2 yield that $J_{p}=J_{p}(\mathbf{R})$ also has the W.A.P. for $1<p<\infty$. More generally, Theorem 5.3 below implies that $J_{p}\left(\mathbf{R}^{n}\right) \approx J_{p} \oplus \ldots \oplus J_{p}$ ( $n$ summands) has the W.A.P. for $n \in \mathbf{N}$. This suggests the following problem (a similar question may obviously be raised for other James-Lindenstrauss-type constructions).

Problem 2.4. Does $J_{p}(E)$ have the W.A.P. whenever $E$ is reflexive and $1<p<$ $\infty$ ?

Recall that a Banach algebra $A$ has a bounded left approximate identity (abbreviated B.L.A.I.) if there is a bounded net $\left(x_{\alpha}\right) \subset A$ such that

$$
\lim _{\alpha}\left\|y-x_{\alpha} y\right\|=0 \quad \text { for } y \in A \text {. }
$$

A bounded right approximate identity (B.R.A.I. for short) in $A$ is obtained by considering $\left\|y-y x_{\alpha}\right\|$ in (2.6). The following observation contains an application of the W.A.P. to algebras of weakly compact operators.

Proposition 2.5. (i) If $E$ has the W.A.P., then the Banach algebra $W(E)$ has a B.L.A.I.

(ii) $W(J)$ has a B.L.A.I.

Proof. (i) Let $U \in W(E)$ and $\varepsilon>0$ be arbitrary. By applying (1.1) to the weakly compact set $\overline{U B_{E}}$ we obtain $V \in W(E)$ satisfying $\|V\| \leq C$ and

$$
\|U-V U\|=\sup _{x \in B_{E}}\|U x-V U x\|<\varepsilon .
$$

Here $C<\infty$ is a uniform constant. It follows that $W(E)$ has a B.L.A.I. by a well-known sufficient condition from BD, Prop. 11.2].

Part (ii) follows from (i) and Theorem 2.2. 
Remarks 2.6. (i) Loy and Willis [LW, Cor. 2.4] established that $W(J)$ has a B.R.A.I. using a different matrix-type argument. Their result suggested the problem of whether $W(J)$ also has a B.L.A.I. This question was not stated in print, but it was known to specialists (R.J. Loy and G.A. Willis, personal communication).

Several authors have studied the existence of bounded left or right approximate identities for algebras of operators, chiefly for closed subalgebras of the compact operators on a Banach space. For such subalgebras the existence of a B.R.A.I. implies the existence of a B.L.A.I. [GW, Cor. 2.7], but no results of this type are known in the setting of $W(E)$. We refer to [D, 2.9.37 and 2.9.67] for further information and references.

(ii) The converse of Proposition 2.5 (i) fails already for $E=\ell^{1}$; see [T1, p. 107].

\section{3. $J^{*}$ HAS THE W.A.P.}

Recall that the dual $J^{*}$ is also quasi-reflexive of order 1 , but that $J^{*}$ is quite different from $J$ as a Banach space. For instance, the norm in $J^{*}$ is not given by any concrete formula, $J^{*}$ does not embed into $J$ by [J2, Thm. 3] and $J$ does not embed into $J^{*}$ by [A, Thm. 7] (see also $[\mathrm{P}$ for a different approach). This provides ample motivation for considering weakly compact approximation in $J^{*}$.

The argument that $J^{*}$ has the W.A.P. follows the basic outline of section 2, but the details are more involved. We recall a few relevant facts about $J^{*}$. Put $f_{n}=\sum_{j=1}^{n} e_{j} \in J$ for $n \in \mathbf{N}$. Then $\left(f_{n}\right)$ is a boundedly complete Schauder basis for $J$. Put $S^{*}(x)=\sum_{s \in S} x(s)$ for $x=\sum_{s=1}^{\infty} x(s) f_{s} \in J$ and any interval $S \subset \mathbf{N}$. In the basis $\left(f_{n}\right)$ the square variation norm (2.1) becomes

$$
\|x\|=\sup _{n ; S_{1}<\ldots<S_{n}}\left(\sum_{j=1}^{n} S_{j}^{*}(x)^{2}\right)^{1 / 2}, \quad x=\sum_{s=1}^{\infty} x(s) f_{s} \in J,
$$

where $S_{1}, \ldots, S_{n}$ are intervals of $\mathbf{N}$ satisfying $\max S_{i}<\min S_{i+1}$ for $i=1, \ldots, n-1$ (the interval $S_{n}$ may be unbounded). We denote this by $S_{1}<S_{2}<\ldots<S_{n}$. Thus $\left\|S^{*}\right\|=1$ whenever $S \subset \mathbf{N}$ is an interval. We will require the fact that

$$
\left\|\sum_{k=1}^{n} c_{k} S_{k}^{*}\right\| \leq\left(\sum_{k=1}^{n}\left|c_{k}\right|^{2}\right)^{1 / 2}
$$

whenever $S_{1}<S_{2}<\ldots<S_{n}$ are intervals of $\mathbf{N}$ and $c_{1}, \ldots, c_{n}$ are scalars. Indeed,

$$
\left|\left\langle\sum_{k=1}^{n} c_{k} S_{k}^{*}, x\right\rangle\right|=\left|\sum_{k=1}^{n} c_{k} S_{k}^{*}(x)\right| \leq\left(\sum_{k=1}^{n}\left|c_{k}\right|^{2}\right)^{1 / 2}\left(\sum_{k=1}^{n} S_{k}^{*}(x)^{2}\right)^{1 / 2} \leq\left(\sum_{k=1}^{n}\left|c_{k}\right|^{2}\right)^{1 / 2}\|x\|
$$

for $x=\sum_{s=1}^{\infty} x(s) f_{s} \in J$.

The sequence $\left(f_{n}^{*}\right)$ of biorthogonal functionals to $\left(f_{n}\right)$ forms a $w^{*}$-basis for $J^{*}$, that is, for any $x^{*} \in J^{*}$ there is a unique scalar sequence $\left(a_{j}\right)=\left(x^{*}\left(f_{j}\right)\right)$ so that $x^{*}=\left(w^{*}\right) \sum_{j=1}^{\infty} a_{j} f_{j}^{*}$ as a $w^{*}$-convergent sum in $J^{*}$. It is known that the limit $\lim _{j \rightarrow \infty} a_{j}$ exists for this $w^{*}$-representation of $x^{*}$. We recall the argument, since we will actually need the more precise quantitative version given below in part (ii).

Lemma 3.1. Let $x^{*}=\left(w^{*}\right) \sum_{j=1}^{\infty} a_{j} f_{j}^{*}$ be the unique $w^{*}$-convergent representation of $x^{*} \in B_{J^{*}}$.

(i) Then $\lim _{j \rightarrow \infty} a_{j}$ exists. 
(ii) There is a uniform constant $C<\infty$ with the following property: Let $\varepsilon>0$ and suppose that for some $k \in \mathbf{N}$ there are indices $p_{1}<q_{1}<p_{2}<q_{2}<\ldots<p_{k}<$ $q_{k}$ with $\left|a_{p_{i}}-a_{q_{i}}\right|>\varepsilon$ for $i=1, \ldots, k$. Then $k \leq \frac{C}{\varepsilon^{2}}$.

Proof. Suppose that there are $\varepsilon>0$ and indices $p_{1}<q_{1}<p_{2}<q_{2}<\ldots<p_{k}<q_{k}$ so that $\left|a_{p_{j}}-a_{q_{j}}\right|>\varepsilon$ for all $j \leq k$. By a result of Casazza, Lin and Lohman (see [CLL, Thm. 16] or [FG, Thms. 2.d.1 and 2.c.9]) the sequence $\left(f_{q_{j}}-f_{p_{j}}\right)_{j=1}^{k}$ is equivalent to the unit vector basis of $\ell_{k}^{2}$ with uniform isomorphism constants independent of $\left(p_{j}\right),\left(q_{j}\right)$ and $k \in \mathbf{N}$. Fix $k$ and consider

$$
x_{k}=\frac{1}{\sqrt{k}} \sum_{j=1}^{k} \theta_{j}\left(f_{q_{j}}-f_{p_{j}}\right) \in J
$$

where the signs $\theta_{1}, \ldots, \theta_{k}$ are chosen so that $\theta_{j}\left(a_{q_{j}}-a_{p_{j}}\right)=\left|a_{p_{j}}-a_{q_{j}}\right|$ for $j=$ $1, \ldots, k$. Thus $\left\|x_{k}\right\| \leq C$, where $C$ is a uniform constant. We get that

$$
C \geq x^{*}\left(x_{k}\right)=\frac{1}{\sqrt{k}} \sum_{j=1}^{k} \theta_{j}\left\langle x^{*}, f_{q_{j}}-f_{p_{j}}\right\rangle=\frac{1}{\sqrt{k}} \sum_{j=1}^{k} \theta_{j}\left(a_{q_{j}}-a_{p_{j}}\right) \geq \varepsilon \sqrt{k},
$$

which proves both (i) and (ii).

Suppose that $x^{*}=\left(w^{*}\right) \sum_{j=1}^{\infty} a_{j} f_{j}^{*} \in J^{*}$ and let $a=\lim _{j} a_{j}$. Put $S_{\infty}=\mathbf{N}$, so that $S_{\infty}^{*}\left(\sum_{j=1}^{\infty} c_{j} f_{j}\right)=\sum_{j} c_{j}$ for $\sum_{j=1}^{\infty} c_{j} f_{j} \in J$. We can write

$$
x^{*}=\sum_{j=1}^{\infty} b_{j} f_{j}^{*}+a S_{\infty}^{*}
$$

where $b_{j} \equiv a_{j}-a \rightarrow 0$ as $j \rightarrow \infty$. In (3.3) the sum $\sum_{j=1}^{\infty} b_{j} f_{j}^{*}$ is norm-convergent in $J^{*}$. We will need the following variant of Lemma 2.1 for weakly compact subsets of $J^{*}$.

Lemma 3.2. Let $D \subset J^{*}$ be a weakly compact set.

(i) Suppose that $\left(x_{n}^{*}\right) \subset D$ is a sequence, where $x_{n}^{*}=\sum_{j=1}^{\infty} a_{j}^{(n)} f_{j}^{*}+a_{\infty}^{(n)} S_{\infty}^{*}$ is written as in (3.3) for $n \in \mathbf{N}$. Then there is a subsequence of $\left(x_{n}^{*}\right)$, still denoted by $\left(x_{n}^{*}\right)$, so that

$$
x_{n}^{*} \stackrel{w}{\longrightarrow} x^{*}=\sum_{j=1}^{\infty} a_{j} f_{j}^{*}+a_{\infty} S_{\infty}^{*} \quad \text { as } n \rightarrow \infty,
$$

where $x^{*}$ is represented as in (3.3) and $\lim _{n \rightarrow \infty} a_{j}^{(n)}=a_{j}$ for all $j \in \mathbf{N} \cup\{\infty\}$.

(ii) For all $n \in \mathbf{N}$ and $\delta>0$ there is $m>n$ so that for all $x^{*}=\sum_{j=1}^{\infty} a_{j} f_{j}^{*}+$ $a_{\infty} S_{\infty}^{*} \in D$ in the representation (3.3), there is $j=j\left(x^{*}\right) \in[n, m)$ satisfying $\left|a_{j}\right|<\delta$.

Proof. (i) The weak compactness of $D$ gives a subsequence of $\left(x_{n}^{*}\right)$, still denoted by $\left(x_{n}^{*}\right)$, so that $x_{n}^{*} \stackrel{w}{\longrightarrow} x^{*}$ as $n \rightarrow \infty$. Write $x^{*}=\sum_{j=1}^{\infty} a_{j} f_{j}^{*}+a_{\infty} S_{\infty}^{*}$ as in (3.3). Let $x^{* *} \in J^{* *}$ satisfy $x^{* *}\left(S_{\infty}^{*}\right)=1$ and $x^{* *}\left(f_{j}^{*}\right)=0$ for all $j \in \mathbf{N}$. Hence $a_{\infty}^{(n)}=x^{* *}\left(x_{n}^{*}\right) \rightarrow x^{* *}\left(x^{*}\right)=a_{\infty}$ as $n \rightarrow \infty$. Moreover, $a_{j}^{(n)}+a_{\infty}^{(n)}=x_{n}^{*}\left(f_{j}\right) \rightarrow$ $x^{*}\left(f_{j}\right)=a_{j}+a_{\infty}$ as $n \rightarrow \infty$ for $j \in \mathbf{N}$. It follows that $\lim _{n \rightarrow \infty} a_{j}^{(n)}=a_{j}$ for each $j \in \mathbf{N}$. 
(ii) Suppose to the contrary that $n \in \mathbf{N}$ and $\delta>0$ are such that for any $m>n$ there is $x_{m}^{*}=\sum_{j=1}^{\infty} a_{j}^{(m)} f_{j}^{*}+a_{\infty}^{(m)} S_{\infty}^{*} \in D$ represented as in (3.3), for which $\left|a_{j}^{(m)}\right| \geq \delta$, for all $j \in[n, m)$. By part (i) there is a subsequence $\left(x_{m}^{*}\right)$ for which

$$
x_{m}^{*} \stackrel{w}{\longrightarrow} x^{*}=\sum_{j=1}^{\infty} a_{j} f_{j}^{*}+a_{\infty} S_{\infty}^{*} \quad \text { as } m \rightarrow \infty,
$$

where $x^{*}$ is written as in (3.3) and $\lim _{m \rightarrow \infty} a_{j}^{(m)}=a_{j}$ for all $j \in \mathbf{N}$. This implies that $\left|a_{j}\right| \geq \delta$ for all $j \geq n$, which contradicts the properties of the expansion (3.3).

We are ready to prove the main result of this section.

Theorem 3.3. $J^{*}$ has the W.A.P.

Proof. Let $D \subset B_{J^{*}}$ be a fixed weakly compact subset and $\varepsilon>0$. The desired approximating operator $V \in W\left(J^{*}\right)$ satisfying (1.1) will again be constructed in several stages.

Step 1. We first fix some notation. Given $n_{1}<\ldots<n_{k}<n_{k+1}$ we define the averaging functionals $B_{\left[n_{j}, n_{j+1}\right)}$ and $B_{\left(n_{1}, \ldots, n_{k+1}\right)}$ on $J^{*}$ by

$$
B_{\left[n_{j}, n_{j+1}\right)}\left(x^{*}\right)=\frac{1}{n_{j+1}-n_{j}} \sum_{s=n_{j}}^{n_{j+1}-1} a_{s}, \quad B_{\left(n_{1}, \ldots, n_{k+1}\right)}\left(x^{*}\right)=\frac{1}{k} \sum_{j=1}^{k} B_{\left[n_{j}, n_{j+1}\right)}\left(x^{*}\right)
$$

for $x^{*}=\sum_{i=1}^{\infty} a_{i} f_{i}^{*}+a_{\infty} S_{\infty}^{*} \in J^{*}$ written as in (3.3) and $j=1, \ldots, k$. Note that $B_{\left[n_{j}, n_{j+1}\right)} \in J^{* *}$ and that $\left\|B_{\left[n_{j}, n_{j+1}\right)}\right\| \leq 2$ for $j \in \mathbf{N}$. In fact, it is easy to see that $\left|a_{s}\right|=\left|x^{*}\left(f_{s}\right)\right|+\left|a_{\infty}\right| \leq 2\left\|x^{*}\right\|$ for $x^{*}=\sum_{i=1}^{\infty} a_{i} f_{i}^{*}+a_{\infty} S_{\infty}^{*} \in J^{*}$ and $s \in \mathbf{N}$.

Let $n \in \mathbf{N}$ and $\delta>0$ be given. By applying Lemma 3.2(ii) repeatedly for $\delta / 2>0$ we find a sequence $n=n_{1}<n_{2}<\ldots$ of $\mathbf{N}$, such that for any $x^{*}=$ $\sum_{i=1}^{\infty} a_{i} f_{i}^{*}+a_{\infty} S_{\infty}^{*} \in D$ and $j \in \mathbf{N}$ there is $i=i\left(x^{*}\right) \in\left[n_{j}, n_{j+1}\right)$ satisfying $\left|a_{i}\right|<\delta / 2$. Here $n_{j+1}=m\left(n_{j}, \delta / 2\right)$ is given by Lemma 3.2(ii). We first verify that sufficiently long double averages $B_{\left(n_{1}, \ldots, n_{k+1}\right)}$ are uniformly small on the weakly compact set $D$.

Claim 1. There is $k \in \mathbf{N}$ such that

$$
\left|B_{\left(n_{1}, \ldots, n_{k+1}\right)}\left(x^{*}\right)\right|<2 \delta \quad \text { for all } x^{*} \in D \text {. }
$$

Proof. The idea resembles that of Claim 1 in Theorem 2.2. Let $x^{*} \in D$ be arbitrary and write $x^{*}=\sum_{i=1}^{\infty} a_{i} f_{i}^{*}+a_{\infty} S_{\infty}^{*}$ as in (3.3). Fix $k \in \mathbf{N}$ and consider

$$
I=\left\{j \leq k:\left|a_{i}\right| \geq \delta \text { for some } i \in\left[n_{j}, n_{j+1}\right)\right\}
$$

(note that $I$ depends on $x^{*}, k$ and $\delta$ ). Write $I=\left\{j_{1}, \ldots, j_{r}\right\}$ and pick $p_{s}, q_{s} \in$ $\left[n_{j_{s}}, n_{j_{s}+1}\right)$ such that $\left|a_{p_{s}}\right| \geq \delta$ and $\left|a_{q_{s}}\right|<\delta / 2$ for $s=1, \ldots, r$. Since $\left|a_{q_{s}}-a_{p_{s}}\right|>$ $\delta / 2$ for each $s=1, \ldots, r$, it follows from Lemma 3.1.(ii) that $r=|I| \leq 4 C / \delta^{2}$ for some uniform constant $C<\infty$. For $j \notin I$ we have $\left|a_{s}\right|<\delta$ for all $s \in\left[n_{j}, n_{j+1}\right)$, so 
that $\left|B_{\left[n_{j}, n_{j+1}\right)}\left(x^{*}\right)\right|<\delta$. We get the estimates

$$
\begin{aligned}
\left|B_{\left(n_{1}, \ldots, n_{k+1}\right)}\left(x^{*}\right)\right| & \leq \frac{1}{k}\left(\sum_{j \in I}\left|B_{\left[n_{j}, n_{j+1}\right)}\left(x^{*}\right)\right|+\sum_{j \notin I}\left|B_{\left[n_{j}, n_{j+1}\right)}\left(x^{*}\right)\right|\right) \\
& \leq \frac{2|I|}{k}+\frac{(k-|I|) \delta}{k} \\
& \leq \frac{8 C}{k \delta^{2}}+\delta<2 \delta
\end{aligned}
$$

for all large enough $k=k(\delta)$.

Step 2. Fix a decreasing positive sequence $\left(\varepsilon_{j}\right)$ such that $\sum_{j=1}^{\infty} \varepsilon_{j}<\varepsilon$. Next apply Claim 1 successively to get a sequence of finite subdivisions $1=n_{r_{1}}<$ $n_{r_{1}+1}<\ldots<n_{r_{2}}<n_{r_{2}+1}<\ldots<n_{r_{3}}<\ldots$ so that

$$
\left|B_{\left(n_{r_{j}}, \ldots, n_{r_{j+1}}\right)}\left(x^{*}\right)\right|<\varepsilon_{j} \text { for all } x^{*} \in D \text { and } j \in \mathbf{N} \text {. }
$$

Put $B_{j}=B_{\left(n_{r_{j}}, \ldots, n_{r_{j+1}}\right)}$ and $I_{j}=\left[n_{r_{j}}, n_{r_{j+1}}\right)$ for $j \in \mathbf{N}$. Define the linear map $V$ on $J^{*}$ by

$$
V x^{*}=V\left(\sum_{i=1}^{\infty} a_{i} f_{i}^{*}+a_{\infty} S_{\infty}^{*}\right)=x^{*}-\sum_{j=1}^{\infty} B_{j}\left(x^{*}\right) I_{j}^{*}, \quad x^{*} \in J^{*} .
$$

We next verify that $V$ is a weakly compact operator on $J^{*}$ that satisfies (1.1) for $D$ and the given $\varepsilon>0$. The uniform bound for $\|V\|$ will require additional tools compared to the argument in section 2.

Claim 2. $\left\|x^{*}-V x^{*}\right\|<\varepsilon$ for all $x^{*} \in D$.

Proof of Claim 2. Recall that $\left\|I_{j}^{*}\right\|=1$ for $j \in \mathbf{N}$ by (3.1). Hence it follows from (3.4) that

$$
\left\|x^{*}-V x^{*}\right\| \leq \sum_{j=1}^{\infty}\left|B_{j}\left(x^{*}\right)\right| \cdot\left\|I_{j}^{*}\right\|<\sum_{j=1}^{\infty} \varepsilon_{j}<\varepsilon, \quad x^{*} \in D .
$$

Claim 3. $\|V\| \leq 7$ (independently of the subdivisions).

Proof of Claim 3. Let $U$ be the linear map

$$
U x^{*}=\sum_{j=1}^{\infty} B_{j}\left(x^{*}\right) I_{j}^{*}, \quad x^{*} \in J^{*} .
$$

We will write $x^{*}=\sum_{s=1}^{\infty} x^{*}(s) f_{s}^{*}+x_{\infty}^{*} S_{\infty}^{*} \in J^{*}$ with respect to the Schauder basis $\left\{f_{j}^{*}: j \in \mathbf{N}\right\} \cup\left\{S_{\infty}^{*}\right\}$ of $J^{*}$. Here $x_{\infty}^{*}=\lim _{s \rightarrow \infty} b_{s}=\lim _{s \rightarrow \infty} x^{*}\left(f_{s}\right)$ in terms of the $w^{*}$-representation $x^{*}=\left(w^{*}\right) \sum_{s=1}^{\infty} b_{s} f_{s}^{*} \in J^{*}$. Thus $\left|x_{\infty}^{*}\right| \leq\left\|x^{*}\right\|$, so that

$$
\left\|\sum_{s=1}^{\infty} x^{*}(s) f_{s}^{*}\right\| \leq\left\|x^{*}\right\|+\left|x_{\infty}^{*}\right| \cdot\left\|S_{\infty}^{*}\right\| \leq 2\left\|x^{*}\right\|
$$

for $x^{*} \in J^{*}$. We next establish that

$$
\left\|U x^{*}\right\| \leq 3
$$

for all finitely supported $x^{*} \in\left[f_{1}^{*}, \ldots, f_{n}^{*}\right] \cap B_{J^{*}}, n \in \mathbf{N}$. Since $\left[f_{1}^{*}, \ldots, f_{n}^{*}\right] \cap B_{J^{*}}$ is the closed convex hull of its extreme points, it is enough to show that (3.6) holds for 
the extreme points $x^{*}$ of $\left[f_{1}^{*}, \ldots, f_{n}^{*}\right] \cap B_{J^{*}}$. Towards this it suffices by Proposition 3.4 below to restrict attention to functionals $x^{*}$ having the special form

$$
x^{*}=\sum_{j=1}^{r} c_{j} S_{j}^{*}, \quad \text { where } \sum_{j=1}^{r}\left|c_{j}\right|^{2}=1 \text { and } S_{1}<\ldots<S_{r}
$$

are intervals of $\mathbf{N}$ with $\max S_{r} \leq n$. Here $n \in \mathbf{N}$ is arbitrary. We fix some more notation for convenience. Put $I_{j, k}=\left[n_{r_{j}+k}, n_{r_{j}+k+1}\right)$ for $k=0, \ldots, r_{j+1}-r_{j}-1$ corresponding to the subdivision $n_{r_{j}}<n_{r_{j}+1}<\ldots<n_{r_{j+1}-1}<n_{r_{j+1}}$ of $I_{j}=$ $\left[n_{r_{j}}, n_{r_{j+1}}\right)$ for $j \in \mathbf{N}$. Thus $\mathbf{N}=\bigcup_{j=1}^{\infty}\left(\bigcup_{k=0}^{r_{j+1}-r_{j}-1} I_{j, k}\right)$ is a partition of $\mathbf{N}$.

Let $x^{*}=\sum_{i=1}^{r} c_{i} S_{i}^{*}$ be as above. We have

$$
U x^{*}=\sum_{j=1}^{\infty} B_{j}\left(x^{*}\right) I_{j}^{*}=\sum_{j=1}^{\infty}\left(\sum_{i=1}^{r} c_{i} B_{j}\left(S_{i}^{*}\right)\right) I_{j}^{*}
$$

by definition. Note first that $B_{j}\left(S_{i}^{*}\right)=1$ if the interval $I_{j}=\left[n_{r_{j}}, n_{r_{j+1}}\right) \subset S_{i}$. Let $A$ consist of the indices $i \in\{1, \ldots, r\}$ such that $I_{j} \subset S_{i}$ for some $j \in \mathbf{N}$, and put $\overline{S_{i}} \equiv \bigcup_{j: I_{j} \subset S_{i}} I_{j}$ for $i \in A$. Note that $\overline{S_{i}}$ is an interval contained in $S_{i}$. Hence the corresponding coordinates satisfy $U x^{*}(s)=c_{i}$ for $s \in \overline{S_{i}}$ and $i \in A$.

Suppose next that $I_{j}=\left[n_{r_{j}}, n_{r_{j+1}}\right)$ is not contained in any of the intervals $S_{1}, \ldots, S_{r}$. For these coordinates $U x^{*}$ looks like

$$
\left(\sum_{i=1}^{r} c_{i} B_{j}\left(S_{i}^{*}\right)\right) I_{j}^{*} \equiv d_{j} I_{j}^{*}
$$

Here

$$
B_{j}\left(S_{i}^{*}\right)=\frac{1}{n_{r_{j+1}}-n_{r_{j}}} \sum_{k=0}^{r_{j+1}-r_{j}-1} \frac{\left|I_{j, k} \cap S_{i}\right|}{\left|I_{j, k}\right|} \in[0,1) .
$$

Note that $d_{j}=\sum_{i=1}^{r} B_{j}\left(S_{i}^{*}\right) c_{i}$ is an "absolutely convex" combination of $c_{1}, \ldots, c_{r}$, since $\sum_{i=1}^{r} B_{j}\left(S_{i}^{*}\right) \leq 1$. Put

$$
E=\left\{j \in \mathbf{N}: I_{j} \not \subseteq S_{i} \text { for any } i=1, \ldots, r\right\} .
$$

Consequently we may coordinatewise split

$$
U x^{*}=\sum_{i \in A} c_{i}\left(\overline{S_{i}}\right)^{*}+\sum_{j \in E_{1}} d_{j} I_{j}^{*}+\sum_{j \in E_{2}} d_{j} I_{j}^{*} \equiv \Sigma_{1}+\Sigma_{2}+\Sigma_{3},
$$

where the above sums are actually finite. Here $E_{1}$ and $E_{2}$ contain every second index of $E$, respectively. It is immediate from (3.2) that $\left\|\Sigma_{1}\right\| \leq\left(\sum_{i \in A}\left|c_{i}\right|^{2}\right)^{1 / 2} \leq 1$.

Suppose that $j \in E_{1}$. By (3.7) one has $\left|d_{j}\right| \leq\left|c_{i(j)}\right| \equiv \max \left\{\left|c_{i}\right|: I_{j} \cap S_{i} \neq \emptyset\right\}$ for a suitable $i(j) \in\{1, \ldots, r\}$ with $I_{j} \cap S_{i(j)} \neq \emptyset$. Since $E_{1}$ contains every second index of $E$ it follows that $i\left(j_{1}\right) \neq i\left(j_{2}\right)$ once $j_{1}, j_{2} \in E_{1}$ and $j_{1} \neq j_{2}$. Thus one gets from (3.2) that

$$
\left\|\Sigma_{2}\right\| \leq\left(\sum_{j \in E_{1}}\left|d_{j}\right|^{2}\right)^{1 / 2} \leq\left(\sum_{i=1}^{r}\left|c_{i}\right|^{2}\right)^{1 / 2}=1 .
$$

In a similar manner one checks that $\left\|\Sigma_{3}\right\| \leq 1$. By putting these estimates together we get $\left\|U x^{*}\right\| \leq 3$ for these particular functionals $x^{*}=\sum_{j=1}^{r} c_{j} S_{j}^{*} \in\left[f_{1}^{*}, \ldots, f_{n}^{*}\right] \cap$ $B_{J^{*}}$, which yields (3.6) (modulo Proposition 3.4 below). 
Finally, from (3.5), (3.6) and $U\left(S_{\infty}^{*}\right)=0$ we obtain by approximation that

$$
\left\|U y^{*}\right\|=\left\|U\left(\sum_{s=1}^{\infty} y^{*}(s) f_{s}^{*}\right)\right\| \leq 3\left\|\sum_{s=1}^{\infty} y^{*}(s) f_{s}^{*}\right\| \leq 6\left\|y^{*}\right\|
$$

for $y^{*}=\sum_{s=1}^{\infty} y^{*}(s) f_{s}^{*}+y_{\infty}^{*} S_{\infty}^{*} \in J^{*}$. We deduce that $\|V\|=\|I-U\| \leq 7$.

Claim 4. $V \in W\left(J^{*}\right)$.

Proof of Claim 4. Let $\left(x_{n}^{*}\right) \subset B_{J^{*}}$ be an arbitrary sequence. We are required to find a subsequence, still denoted by $\left(x_{n}^{*}\right)$, so that $\left(V x_{n}^{*}\right)$ is weakly convergent. Write $x_{n}^{*}=\sum_{j=1}^{\infty} a_{j}^{(n)} f_{j}^{*}+a_{\infty}^{(n)} S_{\infty}^{*}$ as in (3.3) for $n \in \mathbf{N}$. By the $w^{*}$-sequential compactness and a diagonalization we may pass to a subsequence and assume without loss of generality that $\lim _{n \rightarrow \infty} a_{j}^{(n)}=a_{j}$ for $j \in \mathbf{N} \cup\{\infty\}, \lim _{j \rightarrow \infty} a_{j}=a$, and

$$
x_{n}^{*} \stackrel{w^{*}}{\longrightarrow} x^{*} \equiv\left(w^{*}\right) \sum_{j=1}^{\infty} a_{j} f_{j}^{*}+a_{\infty} S_{\infty}^{*}=\sum_{j=1}^{\infty}\left(a_{j}-a\right) f_{j}^{*}+\left(a_{\infty}+a\right) S_{\infty}^{*} \in J^{*}
$$

as $n \rightarrow \infty$. The latter representation is the norm-convergent one as in (3.3). Put

$$
y_{n}^{*}=x_{n}^{*}-a \sum_{j=1}^{n} I_{j}^{*}=\sum_{s=1}^{\max I_{n}}\left(a_{s}^{(n)}-a\right) f_{s}^{*}+\sum_{s=\max I_{n}+1}^{\infty} a_{s}^{(n)} f_{s}^{*}+a_{\infty}^{(n)} S_{\infty}^{*}
$$

as a norm-convergent sum for $n \in \mathbf{N}$. Let $S_{\infty}^{* *}=w^{*}-\lim _{n \rightarrow \infty} f_{n}$ in $J^{* *}$, so that $S_{\infty}^{* *}\left(S_{\infty}^{*}\right)=1$ and $S_{\infty}^{* *}\left(f_{j}^{*}\right)=0$ for $j \in \mathbf{N}$. Put $g_{j}=f_{j}-S_{\infty}^{* *}$ for $j \in \mathbf{N}$. Then $\left\{g_{j}: j \in \mathbf{N}\right\} \cup\left\{S_{\infty}^{* *}\right\}$ is a Schauder basis for $J^{* *}$, for which

$\lim _{n \rightarrow \infty} g_{j}\left(y_{n}^{*}\right)=\lim _{n \rightarrow \infty}\left(a_{j}^{(n)}-a\right)=a_{j}-a \quad(j \in \mathbf{N}), \lim _{n \rightarrow \infty} S_{\infty}^{* *}\left(y_{n}^{*}\right)=\lim _{n \rightarrow \infty} a_{\infty}^{(n)}=a_{\infty}$.

This yields that $y_{n}^{*} \stackrel{w}{\longrightarrow} y^{*} \equiv \sum_{j=1}^{\infty}\left(a_{j}-a\right) f_{j}^{*}+a_{\infty} S_{\infty}^{*}$ (norm-convergent sum) in $J^{*}$ as $n \rightarrow \infty$.

Note further that $V\left(I_{j}^{*}\right)=I_{j}^{*}-\sum_{k=1}^{\infty} B_{k}\left(I_{j}^{*}\right) I_{k}^{*}=0$ for $j \in \mathbf{N}$, since $B_{k}\left(I_{j}^{*}\right)=\delta_{k, j}$ for $j, k \in \mathbf{N}$ by definition. Thus $V x_{n}^{*}=V y_{n}^{*} \stackrel{w}{\longrightarrow} V y^{*}$ in $J^{*}$ as $n \rightarrow \infty$. The proof of Theorem 3.3 will be complete once we have verified the following auxiliary fact that was used in the proof of Claim 3.

Proposition 3.4. Put $J_{n}^{*}=\left[f_{1}^{*}, \ldots, f_{n}^{*}\right] \subset J^{*}$ for $n \in \mathbf{N}$. Then the extreme points of $B_{J_{n}^{*}}$ are contained in the set of elements of the form

$$
\sum_{j=1}^{k} c_{j} S_{j}^{*}, \quad \text { where } \sum_{j=1}^{k}\left|c_{j}\right|^{2}=1 \text { and } S_{1}<\ldots<S_{k}
$$

are intervals of $\mathbf{N}$ with $\max S_{k} \leq n$.

Proof. It follows from (3.1) and (3.2) that

$$
D=\left\{\sum_{j=1}^{k} c_{j} S_{j}^{*}: S_{1}<\ldots<S_{k} \text { intervals, } \max S_{k} \leq n \text { and } \sum_{j=1}^{k}\left|c_{j}\right|^{2}=1\right\} \subset B_{J_{n}^{*}}
$$

is a symmetric 1-norming set for $J_{n}=\left[f_{1}, \ldots, f_{n}\right] \subset J$. Indeed, it is immediate from (3.2) that $\left|\left(\sum_{j=1}^{k} c_{j} S_{j}^{*}\right)(x)\right| \leq\|x\|$ for $\sum_{j=1}^{k} c_{j} S_{j}^{*} \in D$ and $x \in J_{n}$. Conversely, 
for any non-zero $x \in J_{n}$ there are intervals $S_{1}<\ldots<S_{k}$ with $\max S_{k} \leq n$, so that $\|x\|^{2}=\sum_{j=1}^{k} S_{j}^{*}(x)^{2}$. By choosing $c_{j}=\|x\|^{-1} S_{j}^{*}(x)$ for $j=1, \ldots, k$ we get that

$$
\|x\|=\sum_{j=1}^{k} c_{j} S_{j}^{*}(x)=\left(\sum_{j=1}^{k} c_{j} S_{j}^{*}\right)(x)
$$

where $\sum_{j=1}^{k}\left|c_{j}\right|^{2}=1$.

Observe next that $\overline{c o}(D)=B_{J_{n}^{*}}$. In fact, if $\overline{c o}(D) \subsetneq B_{J_{n}^{*}}$, then the Hahn-Banach theorem would give $x_{0}^{*} \in B_{J_{n}^{*}}$ and $x \in B_{J_{n}}$ so that $x_{0}^{*}(x)=1$ and $x^{*}(x) \leq \alpha<$ $1=\|x\|$ for all $x^{*} \in \overline{c o}(D)$. This contradicts the 1-norming property of $D$. Finally, since $B_{J_{n}^{*}}=\overline{c o}(D)$, Milman's "converse" to the Krein-Milman theorem (see e.g. [Ph, Prop. 1.5]) yields that the set of extreme points $\operatorname{ext}\left(B_{J_{n}^{*}}\right) \subset D$.

Proposition 2.5(i) and Theorem 3.3 have the following consequence.

Corollary 3.5. $W\left(J^{*}\right)$ has a B.L.A.I.

Remarks 3.6. (i) Recall that $J^{* *} \approx J$, so that $J^{* * *} \approx J^{*}$. Hence Theorems 2.2 and 3.3 imply that the $k$-th dual $J^{(k)}$ has the W.A.P. for all $k \geq 2$. Moreover, $J=\left[f_{n}^{*}: n \in \mathbf{N}\right]^{*}$, since $\left(f_{n}\right)$ is a monotone boundedly complete basis for $J$. It follows that the predual $J_{*} \equiv\left[f_{n}^{*}: n \in \mathbf{N}\right]$ of $J$ also has the W.A.P., since $J^{*} \approx J_{*} \oplus\left[S_{\infty}^{*}\right]$ and the W.A.P. is inherited by complemented subspaces (cf. Lemma 5.2 (i) below).

(ii) The averaging functionals $B_{j} \in J^{* *}$ employed in the proof of Theorem 3.3 are not $w^{*}$-continuous on $J^{*}$, so that the approximating operators $V \in W\left(J^{*}\right)$ are not adjoints of operators on $J$. Hence Corollary 3.5 does not (by itself) imply the earlier result of $[\mathrm{LW}$, Cor. 2.4] that $W(J)$ has a B.R.A.I.

\section{A family of James-type spaces having the W.A.P.}

The class of quasi-reflexive Banach spaces is extensive, and sections 2 and 3 suggested the question of whether there are quasi-reflexive spaces $E$ that fail to have the W.A.P. During the course of this work Argyros and Tolias discovered that a class of hereditarily indecomposable (H.I.) spaces constructed recently (for different purposes) in $\mathrm{ArT}$ contains quasi-reflexive spaces of this kind. We refer to ArT for the description of these spaces, and to ArT, Prop. 14.10] for the details of the following example.

Example 4.1. There is a quasi-reflexive H.I. space $E$ that fails to have the W.A.P.

One reason for such spaces $E$ to fail the W.A.P. appears to be that they admit "few" weakly compact operators in the sense that

$$
L(E)=\{\lambda I+V: \lambda \in \mathbf{C}, V \text { is strictly singular and weakly compact }\} .
$$

In contrast the quasi-reflexive spaces studied in this paper have many weakly compact subsets, but they are also sufficiently rich in weakly compact operators to suggest that they may have the W.A.P. The purpose of this section is to extend the results of section 2 to a general class of quasi-reflexive spaces considered by Bellenot, Haydon and Odell [BHO]. This class also contains $J$, but the reader is expected to already be familiar with the argument from section 2 . The desired approximating operators will also be somewhat more involved in the general case. 
Let $\left(h_{j}\right)$ be a normalized Schauder basis for a reflexive space $E$. The Banach space $J\left(h_{j}\right)$ consists of the scalar sequences $\left(a_{j}\right)$ so that $\lim _{j \rightarrow \infty} a_{j}=0$ and

$$
\left\|\left(a_{j}\right)\right\|=\sup \left\{\left\|\sum_{j=1}^{n}\left(a_{p_{j}}-a_{q_{j}}\right) h_{p_{j}}\right\|: 1 \leq p_{1}<q_{1}<\ldots<p_{n}<q_{n}, n \in \mathbf{N}\right\}<\infty .
$$

We obtain $J$ with an equivalent norm to $(2.1)$, if $\left(h_{j}\right)$ is the standard coordinate basis of $\ell^{2}$. The reference [BHO] contains the basic information about this construction, where it is discussed in terms the boundedly complete basis (analogous to $(3.1)$ for $J)$. All these spaces $J\left(h_{j}\right)$ are quasi-reflexive of order 1 by $\mathrm{BHO}$. Thm. 4.1]. In addition, $J\left(h_{j}\right) \approx J\left(u_{j}\right) \approx J\left(g_{j}\right)$ [BHO Prop. 1.1], where $\left(u_{j}\right)$ is the unconditionalization of $\left(h_{j}\right)$ defined by

$$
\left\|\sum_{j=1}^{\infty} a_{j} u_{j}\right\|=\sup \left\{\left\|\sum_{j=1}^{\infty} \theta_{j} a_{j} h_{j}\right\|:\left(\theta_{j}\right) \in\{-1,1\}^{\mathbf{N}}\right\}<\infty
$$

and $\left(g_{j}\right)$ is the right dominant version of $\left(h_{j}\right)$ (or of $\left(u_{j}\right)$ ) given by

$$
\left\|\sum_{j=1}^{\infty} a_{j} g_{j}\right\|=\sup \left\{\left\|\sum_{j=1}^{\infty} a_{n(i)} h_{m(i)}\right\|: 1 \leq m(1) \leq n(1)<m(2) \leq n(2)<\ldots\right\}<\infty .
$$

Hence we may and will assume in the sequel that the original basis $\left(h_{j}\right)$ of $E$ is 1-unconditional, and in view of [BHO] Prop. 1.1.(4)] that

$$
\left\|\sum_{i=1}^{\infty} a_{n(i)} h_{m(i)}\right\| \leq 2\left\|\sum_{i=1}^{\infty} a_{n(i)} h_{n(i)}\right\|
$$

for all $\sum_{j=1}^{\infty} a_{j} h_{j} \in E$ and all sequences $1 \leq m(1) \leq n(1)<\ldots<m(i) \leq n(i)<\ldots$.

Let $\left(e_{j}\right)$ stand for the unit vector basis in $J\left(h_{j}\right)$, which is a normalized monotone Schauder basis for $J\left(h_{j}\right)$. Recall that the basic sequence $\left(x_{j}\right)$ in $J\left(h_{j}\right)$ is a skipped block basic sequence of $\left(e_{j}\right)$ if for all $j$ there is $n(j) \in \mathbf{N}$ so that max $\operatorname{supp}\left(x_{j}\right)<$ $n(j)<\min \operatorname{supp}\left(x_{j+1}\right)$. Here, as well as in the sequel, the support $\operatorname{supp}(x)$ of $x \in J\left(h_{j}\right)$ is with respect to the basis $\left(e_{j}\right)$. Every normalized skipped block basic sequence $\left(x_{n}\right)$ of $\left(e_{j}\right)$ is $C$-unconditional in $J\left(h_{j}\right)$ with a uniform constant $C<\infty$; see [BHO, Prop. 2.1.(2)].

We will require some additional tools and auxiliary results in order to extend the argument of section 2 to the present setting. Our first result concerns the uniform unconditionality of skipped block sequences of a special type in $J\left(h_{j}\right)$. It is convenient to denote the natural projection of $J\left(h_{j}\right)$ onto the span $\left[e_{s}: m \leq s<n\right]$ by $P_{[m, n)}$, that is, $P_{[m, n)}=P_{n-1}\left(I-P_{m-1}\right)$ for $2 \leq m<n$. Here $\left(P_{n}\right)$ are the basis projections on $J\left(h_{j}\right)$ with respect to $\left(e_{j}\right)$. Thus $\left\|P_{[m, n)}\right\| \leq 2$ for $m \geq 2$.

Lemma 4.2. Let $\left(x_{j}\right)$ be a skipped block basic sequence of $\left(e_{j}\right)$ and let $S_{j} \subset \mathbf{N}$ be the smallest intervals satisfying $S_{j} \supset \operatorname{supp}\left(x_{j}\right)$ for $j \in \mathbf{N}$. Suppose that $\left(n_{j}\right) \subset \mathbf{N}$ is an increasing sequence so that $n_{0}=1$ and $n_{j} \in S_{j+1}$ for $j \in \mathbf{N}$, and put

$$
y_{j}=P_{\left[n_{j-1}, n_{j}\right)}\left(x_{j}+x_{j+1}\right), \quad j \in \mathbf{N} .
$$


Then there is an absolute constant $C<\infty$ so that

$$
\left\|\sum_{j \in B} a_{j} y_{j}\right\| \leq C\left\|\sum_{j=1}^{\infty} a_{j} y_{j}\right\|
$$

for all subsets $B \subset \mathbf{N}$ and all norm convergent $\sum_{j=1}^{\infty} a_{j} y_{j} \in J\left(h_{i}\right)$.

Proof. Let $B \subset \mathbf{N}$ be a given set. By approximation it is enough to establish (4.3) with a uniform constant $C$ for all finitely supported sums $\sum_{j} a_{j} y_{j}$. Put $z=\sum_{j \in B} a_{j} y_{j}$ and $\tilde{z}=\sum_{j} a_{j} y_{j}$. Suppose that $p_{1}<q_{1}<\ldots<p_{m}<q_{m}$ is a sequence of coordinates norming $z$, so that

$$
\|z\|=\left\|\sum_{j=1}^{m}\left(z\left(p_{j}\right)-z\left(q_{j}\right)\right) h_{p_{j}}\right\|=\left\|\sum_{j=1}^{m} b_{j} h_{p_{j}}\right\|,
$$

where we put $b_{j}=z\left(p_{j}\right)-z\left(q_{j}\right) \neq 0$ for $j=1, \ldots, m$. Here we use the convenient notation $y=\sum_{s=1}^{\infty} y(s) e_{s}$ for elements $y \in J\left(h_{j}\right)$.

Let $T_{r} \subset \mathbf{N}$ be the smallest interval satisfying $T_{r} \supset \operatorname{supp}\left(y_{r}\right)$ for $r \in \mathbf{N}$. We put $E=\left\{j \leq m: p_{j}, q_{j} \in T_{r}\right.$ for some $\left.r\right\}$ and $F=\left\{j \leq m: p_{j} \in T_{r}, q_{j} \in T_{s}\right.$ for $r<s\}$. We initially split

$$
\left\|\sum_{j=1}^{m} b_{j} h_{p_{j}}\right\| \leq\left\|\sum_{j \in E} b_{j} h_{p_{j}}\right\|+\left\|\sum_{j \in F_{1}} b_{j} h_{p_{j}}\right\|+\left\|\sum_{j \in F_{2}} b_{j} h_{p_{j}}\right\| \equiv \Sigma_{1}+\Sigma_{2}+\Sigma_{3} .
$$

Here $F=F_{1} \cup F_{2}$ is the partition of $F$ into every second index. Observe first that $\tilde{b}_{j} \equiv \tilde{z}\left(p_{j}\right)-\tilde{z}\left(q_{j}\right)=z\left(p_{j}\right)-z\left(q_{j}\right)=b_{j}$ whenever $j \in E$, so that $\Sigma_{1} \leq\|\tilde{z}\|$ by definition.

We next verify that $\Sigma_{2} \leq 2\|\tilde{z}\|$. Let $j \in F_{1}$. There are three cases to consider:

$$
\text { (i) } p_{j} \in T_{r}, q_{j} \in T_{s}, \quad \text { (ii) } p_{j} \in T_{s}, q_{j} \in T_{t}, \quad \text { (iii) } p_{j} \in T_{r}, q_{j} \in T_{t} \text {, }
$$

where $r<s<t, r, t \in B$ and $s \notin B$. In case (i) from (4.5) one has $b_{j}=z\left(p_{j}\right)-$ $z\left(q_{j}\right)=z\left(p_{j}\right)$. Since $\left(x_{s}\right)$ is a skipped block sequence, and $y_{s}=P_{\left[n_{s-1}, n_{s}\right)}\left(x_{s}+x_{s+1}\right)$, there is some index $r_{j} \in T_{s}$ for which $y_{s}\left(r_{j}\right)=0$. Hence we may move $q_{j}$ to $r_{j}$, so that the difference

$$
b_{j}=z\left(p_{j}\right)=\tilde{z}\left(p_{j}\right)-\tilde{z}\left(r_{j}\right) \equiv \tilde{b}_{j}
$$

can still be used towards computing $\|\tilde{z}\|$ as in definition (4.1). For case (ii) from (4.5) observe first that $b_{j}=z\left(p_{j}\right)-z\left(q_{j}\right)=-z\left(q_{j}\right)$. There are two possibilities to consider. If $\left|\tilde{z}\left(p_{j}\right)-\tilde{z}\left(q_{j}\right)\right|>\frac{\left|b_{j}\right|}{2}$, then we keep the coordinates $p_{j}<q_{j}$ towards computing $\|\tilde{z}\|$ as in (4.1). In the opposite case, where $\left|\tilde{z}\left(p_{j}\right)-\tilde{z}\left(q_{j}\right)\right| \leq \frac{\left|b_{j}\right|}{2}$, we move the coordinate $q_{j}$ to some $r_{j} \in T_{t}$ satisfying $y_{t}\left(r_{j}\right)=0$. This implies that

$$
\left|\tilde{z}\left(p_{j}\right)-\tilde{z}\left(r_{j}\right)\right|=\left|\tilde{z}\left(p_{j}\right)\right| \geq\left|z\left(q_{j}\right)\right|-\left|z\left(q_{j}\right)-\tilde{z}\left(q_{j}\right)\right|=\left|b_{j}\right|-\left|\tilde{z}\left(q_{j}\right)-\tilde{z}\left(q_{j}\right)\right| \geq \frac{\left|b_{j}\right|}{2} .
$$

Finally, in case (iii) we have $z\left(p_{j}\right)-z\left(q_{j}\right)=\tilde{z}\left(p_{j}\right)-\tilde{z}\left(q_{j}\right)$ (because $r, t \in B$ ), and we retain the pair $p_{j}<q_{j}$.

We get in all cases from (4.5) that $\left|b_{j}\right| \leq 2\left|\tilde{b}_{j}\right|$, where $\tilde{b}_{j} \equiv \tilde{z}\left(p_{j}\right)-\tilde{z}\left(\tilde{q}_{j}\right)$ and $\tilde{q}_{j}$ stands for either $q_{j}$ or $r_{j}$, depending on the indicated choices. The 1unconditionality of the basis $\left(h_{i}\right)$ in $E$ then yields that

$$
\Sigma_{2}=\left\|\sum_{j \in F_{1}} b_{j} h_{p_{j}}\right\| \leq 2\left\|\sum_{j \in F_{1}} \tilde{b}_{j} h_{p_{j}}\right\| \leq 2\|\tilde{z}\|,
$$


since the sequence of pairs $p_{j}<\tilde{q}_{j}$ with $j \in F_{1}$ are admissible coordinates towards computing $\|\tilde{z}\|$ as in (4.1). Indeed, the fact that $F_{1}$ contains every second index of $F$ ensures that the order is preserved in the new sequence if (some of) the coordinates $q_{j}$ are moved.

The estimate $\Sigma_{3} \leq 2\|\tilde{z}\|$ is similar. This completes the proof of (4.3).

The following combinatorial lemma due to Ptak [Pt] (see also [BHO] for the present formulation) will be a crucial tool towards building certain convex combinations of averaging functionals, which will replace the double averages used in sections 2 and 3 . It is convenient to put $\left(\alpha_{1}, \ldots, \alpha_{k}\right) \in\left(S_{\ell_{1}^{k}}\right)_{+}$provided $\sum_{r=1}^{k} \alpha_{r}=1$ and $\alpha_{r} \geq 0$ for $r=1, \ldots, k$.

Lemma 4.3 ([BHO, Lemma 3.1]). Let $0<\delta<1$ be fixed. Suppose that $\mathcal{F}$ is a collection of non-empty finite subsets of $\mathbf{N}$ satisfying the following properties:

(i) $B \in \mathcal{F}$ whenever $\emptyset \neq B \subset A$ and $A \in \mathcal{F}$.

(ii) For every $k \in \mathbf{N}$ and every convex combination $\left(\alpha_{1}, \ldots, \alpha_{k}\right) \in\left(S_{\ell_{1}^{k}}\right)_{+}$, there is $A \in \mathcal{F}$ so that $\sum_{j \in A} \alpha_{j} \geq \delta$.

Then there is an infinite subset $M \subset \mathbf{N}$ so that $A \in \mathcal{F}$ for all non-empty finite sets $A \subset M$.

We are now ready to prove the main result of this section, which establishes the weakly compact approximation property for the James-like spaces $J\left(h_{j}\right)$. In the proof we will denote $x \in J\left(h_{j}\right)$ by $x=\sum_{s=1}^{\infty} x(s) e_{s}$.

Theorem 4.4. Let $\left(h_{j}\right)$ be a normalized Schauder basis for a reflexive Banach space $E$. Then the quasi-reflexive space $J\left(h_{j}\right)$ has the W.A.P.

Proof. Recall that in view of [BHO, Prop. 1.1] we may assume that the Schauder basis $\left(h_{j}\right)$ for $E$ is 1-unconditional and satisfies the right dominance property (4.2). Let $D \subset B_{J\left(h_{j}\right)}$ be a weakly compact set and let $\varepsilon>0$. We again split the argument into distinct steps.

Step 1. Let $0<\delta<1$ and $n \in \mathbf{N}$ be given. By successive applications of Lemma 2.1 we fix a sequence $n=n_{1}<n_{2}<\ldots$ in $\mathbf{N}$, so that for every $x=\sum_{s=1}^{\infty} x(s) e_{s} \in D$ and $j \in \mathbf{N}$ there is some index $s_{j} \in\left[n_{j}, n_{j+1}\right)$ for which $\left|x\left(s_{j}\right)\right|<\frac{\delta}{2^{j+3}}$.

For technical purposes we need to improve this fact by a slight perturbation.

Claim 1. For every $x \in D$ there is a perturbation $x \approx x_{0}+\sum_{j=1}^{\infty} a_{j} y_{j}$ satisfying the following properties:

(i) $x_{0}=P_{\left[1, n_{1}\right)}(x), y_{j} \in\left[e_{s}: n_{j} \leq s<n_{j+1}\right],\left\|y_{j}\right\|=1$ and $a_{j} \geq 0$ for $j \in \mathbf{N}$,

(ii) $\left\|x-\left(x_{0}+\sum_{j=1}^{\infty} a_{j} y_{j}\right)\right\|<\frac{\delta}{4}$ and $\left\|\sum_{j=1}^{\infty} a_{j} y_{j}\right\|<2+\frac{\delta}{4}<3$,

(iii) for every $j \in \mathbf{N}$ one has $y_{j}\left(s_{j}\right)=0$ for some $s_{j} \in\left[n_{j}, n_{j+1}\right)$.

Proof of Claim 1. Let $x=\sum_{s=1}^{\infty} x(s) e_{s} \in D$. We put $x_{0}=P_{\left[1, n_{1}\right)}(x)$ and

$$
v_{j}=\sum_{s \in\left[n_{j}, n_{j+1}\right) ; s \neq s_{j}} x(s) e_{s} \in\left[e_{s}: n_{j} \leq s<n_{j+1}, s \neq s_{j}\right], \quad j \in \mathbf{N} .
$$

Consider $y=x_{0}+\sum_{j=1}^{\infty} v_{j}=x_{0}+\sum_{j=1}^{\infty} a_{j} y_{j}$, where $a_{j}=\left\|v_{j}\right\|$ and $y_{j}=\frac{v_{j}}{\left\|v_{j}\right\|}$ (with obvious modifications if $v_{j}=0$ ). Let $z_{j}=P_{\left[n_{j}, n_{j+1}\right)}(x)$, so that $\left\|z_{j}-v_{j}\right\|=$ $\left|x\left(s_{j}\right)\right|<\frac{\delta}{2^{j+3}}$ for $j \in \mathbf{N}$. Hence $\|x-y\| \leq \sum_{j=1}^{\infty}\left\|z_{j}-v_{j}\right\|<\frac{\delta}{4}$. The other conditions from Claim 1 are satisfied by construction. 
We note that in Claim 1 we also get that

$$
a_{j} \leq\left\|P_{\left[n_{j}, n_{j+1}\right)}(x)\right\|+\left\|z_{j}-v_{j}\right\|<2+\frac{\delta}{2^{j+3}}<3, \quad j \in \mathbf{N} .
$$

Step 2. Define the averaging functionals $A_{j}$ on $J\left(h_{i}\right)$ by

$$
A_{j}(x)=\frac{1}{n_{j+1}-n_{j}} \sum_{s=n_{j}}^{n_{j+1}-1} x(s) \quad \text { for } x=\sum_{s=1}^{\infty} x(s) e_{s} \in J\left(h_{j}\right)
$$

and $j \in \mathbf{N}$. Thus $\left\|A_{j}\right\|=1$ for $j \in \mathbf{N}$, since $|x(s)| \leq\|x\|$ for $x \in J\left(h_{j}\right)$ and $s \in \mathbf{N}$. For any given $k \in \mathbf{N}$ and $\left(\alpha_{1}, \ldots, \alpha_{k}\right) \in\left(S_{\ell_{1}^{k}}\right)+$ we introduce the convex combination

$$
A(x)=\sum_{j=1}^{k} \alpha_{j} A_{j}(x), \quad x \in J\left(h_{j}\right)
$$

By definition $A(x)$ is a convex combination of the coordinates $\left\{x(s): s=n_{1}, \ldots\right.$, $\left.n_{k+1}-1\right\}$ of $x=\sum_{s=1}^{\infty} x(s) e_{s} \in J\left(h_{j}\right)$, and $\|A\| \leq 1$. Note that $A$ depends on $\left(n_{j}\right)$, $k$ and $\left(\alpha_{1}, \ldots, \alpha_{k}\right)$, but our notation does not make this explicit for simplicity.

The double averages involved in the weakly compact approximating operators from sections 2 and 3 relied implicitly on the square variation norm for $J$. Our next aim is to show (see Claim 2 below) that we may choose $k \in \mathbf{N}$ and a convex combination $\left(\alpha_{1}, \ldots, \alpha_{k}\right) \in\left(S_{\ell_{1}^{k}}\right)_{+}$, so that the corresponding $A$ from (4.7) satisfies $|A(x)|<\delta$ for every $x \in D$. For this end Lemma 4.3 will be crucial. We formulate the main technical step here as a separate lemma.

Lemma 4.5. There is an integer $k \in \mathbf{N}$ and a convex combination $\left(\alpha_{1}, \ldots, \alpha_{k}\right) \in$ $\left(S_{\ell_{1}^{k}}\right)_{+}$so that for all $x \in D$ and perturbations $x \approx x_{0}+\sum_{i=1}^{\infty} a_{i} y_{i}$ as in Claim 1 , one has

$$
\sum_{i=1}^{k} \alpha_{i} a_{i}<\frac{\delta}{4}
$$

Proof of Lemma 4.5. Assume to the contrary that for every $k \in \mathbf{N}$ and every convex combination $\left(\alpha_{1}, \ldots, \alpha_{k}\right) \in\left(S_{\ell_{1}^{k}}\right)_{+}$we may find an element $x \in D$ and a perturbation $x \approx x_{0}+\sum_{j=1}^{\infty} a_{j} y_{j}$ satisfying conditions (i)-(iii) of Claim 1 and (4.6), so that

$$
\sum_{j=1}^{k} \alpha_{j} a_{j} \geq \frac{\delta}{4}
$$

We wish to apply the combinatorial Lemma 4.3 to this setting. Let $\mathcal{F}$ be the collection of finite sets

$$
\begin{aligned}
A=\left\{i: a_{i} \geq\right. & \frac{\delta}{8}, \text { where } x_{0}+\sum_{j=1}^{\infty} a_{j} y_{j} \text { satisfies (i), (ii), (iii) of Claim 1, (4.6), } \\
& \text { and (4.9) holds for some } \left.k \text { and }\left(\alpha_{1}, \ldots, \alpha_{k}\right) \in\left(S_{\ell_{1}^{k}}\right)_{+}\right\} .
\end{aligned}
$$

The family $\mathcal{F}$ satisfies the conditions of Lemma 4.3. Indeed,

$$
3 \cdot \sum_{i \in A} \alpha_{i} \geq \sum_{i \in A} \alpha_{i} a_{i} \geq \frac{\delta}{4}-\sum_{i \in A^{c}} \alpha_{i} a_{i}>\frac{\delta}{8}
$$

since $\sum_{i \in A^{c}} \alpha_{i} a_{i}<\frac{\delta}{8}$. Here $A^{c}=\{1, \ldots, k\} \backslash A$. 
Lemma 4.3 yields an infinite set $M=\left\{m_{i}: i \in \mathbf{N}\right\} \subset \mathbf{N}$ for which $A \in \mathcal{F}$ for all finite subsets $A \subset M$. By applying this fact successively to $A_{n}=\left\{m_{1}, \ldots, m_{n}\right\}$ for $n \in \mathbf{N}$, we obtain a sequence of elements $z_{n}=x_{0}^{(n)}+\sum_{j=1}^{\infty} a_{j}^{(n)} y_{j}^{(n)} \in J\left(h_{j}\right)$ satisfying conditions (i)-(iii) of Claim 1 and (4.6), and where further

$$
a_{m_{i}}^{(n)} \geq \frac{\delta}{8} \quad \text { for } i=1, \ldots, n .
$$

By a compactness argument we may pass to a subsequence of $\left(z_{n}\right)$, so that $a_{i}^{(n)} \rightarrow a_{i}$, $y_{i}^{(n)} \rightarrow v_{i}$ and $x_{0}^{(n)} \rightarrow v$ in norm for every $i \in \mathbf{N}$ as $n \rightarrow \infty$. Here the supports satisfy $\operatorname{supp}(v) \subset\left[1, n_{1}\right), \operatorname{supp}\left(v_{i}\right) \subset\left[n_{i}, n_{i+1}\right)$, and by condition (iii) we may further ensure that $v_{i}\left(s_{i}\right)=0$ for some $s_{i} \in\left[n_{i}, n_{i+1}\right)$ for $i \in \mathbf{N}$.

Lemma 4.2 implies that the sequence $\left(v_{i}\right)$ is a $C$-unconditional basic sequence in $J\left(h_{j}\right)$ for some uniform constant $C$. Hence, by passing to the limit above we get that

$$
\left\|\sum_{i=1}^{m} a_{i} v_{i}\right\| \leq 3 C
$$

for $m \in \mathbf{N}$. It follows from (4.11) that $\left(v_{j}\right)$ cannot be a boundedly complete basis for $\left[v_{i}: i \in \mathbf{N}\right]$, since $a_{m_{i}} \geq \frac{\delta}{8}$ for $i \in \mathbf{N}$ by (4.10). Hence [LT 1.c.10] implies that the quasi-reflexive space $J\left(h_{j}\right)$ must contain an isomorphic copy of $c_{0}$. This contradiction completes the proof of Lemma 4.5.

Claim 2. There is $k \in \mathbf{N}$ and $\left(\alpha_{1}, \ldots, \alpha_{k}\right) \in\left(S_{\ell_{1}^{k}}\right)_{+}$so that the corresponding convex combination $A=\sum_{j=1}^{k} \alpha_{j} A_{j} \in J\left(h_{j}\right)^{*}$ given by (4.7) satisfies

$$
|A(x)|<\delta \quad \text { for } x \in D \text {. }
$$

Proof of Claim 2. Let $x \in D$. According to Step 1 we may fix a perturbation $x \approx x_{0}+\sum_{i=1}^{\infty} a_{i} y_{i}$ satisfying conditions (i)-(iii) of Claim 1 . Since $A_{j}\left(x_{0}\right)=0$ by definition, we get that

$$
\begin{aligned}
\left|A_{j}(x)\right| & \leq\left|A_{j}\left(x_{0}+\sum_{i=1}^{\infty} a_{i} y_{i}\right)\right|+\left|A_{j}\left(x-\left(x_{0}+\sum_{i=1}^{\infty} a_{i} y_{i}\right)\right)\right| \\
& \leq\left|a_{j} A_{j}\left(y_{j}\right)\right|+2\left\|x-\left(x_{0}+\sum_{i=1}^{\infty} a_{i} y_{i}\right)\right\| \leq a_{j}+\frac{\delta}{2}
\end{aligned}
$$

for $j \in \mathbf{N}$.

Next we use Lemma 4.5 to find $k \in \mathbf{N}$ and $\left(\alpha_{1}, \ldots, \alpha_{k}\right) \in\left(S_{\ell_{1}^{k}}\right)_{+}$so that (4.8) holds. Then the above estimate implies that

$$
|A(x)| \leq \sum_{j=1}^{k} \alpha_{j}\left|A_{j}(x)\right| \leq \sum_{j=1}^{k} \alpha_{j} a_{j}+\frac{\delta}{2} \cdot \sum_{j=1}^{k} \alpha_{j}<\delta .
$$

This completes the proof of Claim 2 .

Step 3. Fix a positive decreasing sequence $\left(\varepsilon_{i}\right)$ so that $\sum_{i=1}^{\infty} \varepsilon_{i}<\varepsilon / 2$. By applying Steps 1 and 2 successively we get a partition $\mathbf{N}=\bigcup_{j=1}^{\infty} I_{j}$ into successive intervals and a sequence of functionals $\left(V_{j}\right) \subset J\left(h_{j}\right)^{*}$ that satisfy the following properties for $j \in \mathbf{N}$ :

(iv) $\left|V_{j}(x)\right|<\varepsilon_{j} / 2$ for $x \in D$,

(v) $V_{j}$ is a convex combination of the type (4.7) of averages corresponding to some partition of $I_{j}$ into successive subintervals, 
(vi) $V_{j}(x)=V_{j}\left(\sum_{s \in I_{j}} x(s) e_{s}\right)$ for $x=\sum_{s=1}^{\infty} x(s) e_{s} \in J\left(h_{i}\right)$.

Write $I_{j}=\left[t_{j}, t_{j+1}\right)$ for $j \in \mathbf{N}$, where $t_{1}=1$. We put

$$
s_{0}=e_{1}, \quad s_{1}=\sum_{s=2}^{t_{2}-1} e_{s}, \quad s_{j}=\sum_{s=t_{j}}^{t_{j+1}-1} e_{s} \quad \text { for } j \geq 2 .
$$

Define the linear map $\tilde{V}$ on $J\left(h_{j}\right)$ by

$$
\tilde{V} x=\sum_{j=1}^{\infty} V_{j}(x) s_{j-1} \quad \text { for } x \in J\left(h_{j}\right) .
$$

Note that definition (4.12) introduces an additional left "shift" on $J\left(h_{j}\right)$ compared to the arguments in sections 2 and 3. It is immediate that $V=I-\tilde{V}$ satisfies

$$
\|x-V x\|=\left\|\sum_{j=1}^{\infty} V_{j}(x) s_{j-1}\right\| \leq 2 \sum_{j=1}^{\infty}\left|V_{j}(x)\right|<\varepsilon \quad \text { for every } x \in D .
$$

We verify below in Claims 3 and 4 that $V=I-\tilde{V}$ is the desired weakly compact approximating operator on $J\left(h_{j}\right)$. The right dominance property (4.2) will be essential towards getting a uniform bound for $\|\tilde{V}\|$.

Claim 3. $\|V\| \leq 5$ (independently of the subdivisions).

Proof of Claim 3. We estimate $\|\tilde{V}\|$. Let $x=\sum_{s=1}^{\infty} x(s) e_{s} \in J\left(h_{j}\right)$ be finitely supported, and suppose that $\operatorname{supp}(x) \subset \bigcup_{j=1}^{n} I_{j}$ for some $n \in \mathbf{N}$. Let $k_{1}<l_{1}<$ $\ldots<k_{r}<l_{r}$ be a sequence of coordinates that norms $\tilde{V} x$ according to (4.1), so that

$$
\|\tilde{V} x\|=\left\|\sum_{j=1}^{r} a_{j} h_{k_{j}}\right\|,
$$

where $a_{j}=\tilde{V} x\left(k_{j}\right)-\tilde{V} x\left(l_{j}\right)$ for $j=1, \ldots, r$. Since $\left(h_{j}\right)$ is a 1 -unconditional basis of $E$, we may assume that $a_{j} \neq 0$ for $j=1, \ldots, r$.

By conditions (v) and (vi) the element $\tilde{V} x \in J\left(h_{j}\right)$ is constant on each interval $I_{j}$ with $j \geq 2$. In addition, $\tilde{V} x(1)=V_{1}(x)$ and $\tilde{V} x(s)=V_{2}(x)$ for $2 \leq s<t_{2}$. Hence we may assume without loss of generality that no pair $k_{i}$ and $l_{i}$ belongs to the same interval $\operatorname{supp}\left(s_{j}\right)$ for $i=1, \ldots, r$, and that $r \leq n$.

We claim that

$$
\left\|\sum_{j} a_{2 j+1} h_{k_{2 j+1}}\right\| \leq 2\|x\| .
$$

From condition (vi) we get that

$$
a_{2 j+1}=\tilde{V} x\left(k_{2 j+1}\right)-\tilde{V} x\left(l_{2 j+1}\right)=V_{p}(x)-V_{q}(x)
$$

for some $k_{2 j+1} \leq p<q$. By condition (v) we know that $V_{p}(x)$ is a convex combination of the coordinates $x(s)$ with $s \in I_{p}$, and similarly for $V_{q}(x)$ with respect to $I_{q}$. Hence there are coordinates $m_{2 j+1} \in I_{p}$ and $n_{2 j+1} \in I_{q}$, so that

$$
b_{2 j+1} \equiv\left|x\left(m_{2 j+1}\right)-x\left(n_{2 j+1}\right)\right| \geq\left|a_{2 j+1}\right|
$$

for each $j$. Here $k_{1} \leq m_{1}<k_{3} \leq m_{3}<\ldots$, and it is easy to convince oneself that $m_{1}<n_{1}<m_{3}<n_{3}<\ldots$. Thus we get from (4.1), (4.2) and the 1-unconditionality of $\left(h_{j}\right)$ in $E$ that

$$
\left\|\sum_{j} a_{2 j+1} h_{k_{2 j+1}}\right\| \leq\left\|\sum_{j}\left|a_{2 j+1}\right| h_{k_{2 j+1}}\right\| \leq 2\left\|\sum_{j} b_{2 j+1} h_{m_{2 j+1}}\right\| \leq 2\|x\| .
$$


In a similar fashion one has $\left\|\sum_{j} a_{2 j} h_{k_{2 j}}\right\| \leq 2\|x\|$, so that $\|\tilde{V} x\| \leq 4\|x\|$. Consequently $\|V\| \leq 1+\|\tilde{V}\| \leq 5$, which completes the proof of Claim 3 .

Claim 4. $\quad V=I-\tilde{V} \in W\left(J\left(h_{j}\right)\right)$.

Proof of Claim 4. It suffices to verify that $\left(V\left(\sum_{j=0}^{n+1} s_{j}\right)\right)=\left((I-\tilde{V})\left(\sum_{j=0}^{n+1} s_{j}\right)\right)$ is a weak-null sequence in $J\left(h_{j}\right)$, since $\sum_{j=0}^{n+1} s_{j} \stackrel{w^{*}}{\longrightarrow} \mathbf{1}$ as $n \rightarrow \infty$ in $J\left(h_{j}\right)^{* *}$. Recall for this that $J\left(h_{j}\right)^{* *}=J\left(h_{j}\right) \oplus[\mathbf{1}]$, where $\mathbf{1}=(1,1,1, \ldots)$, and that an operator $U \in W\left(J\left(h_{j}\right)\right)$ if and only if $U^{* *}(\mathbf{1}) \in J\left(h_{j}\right)$.

Note first that $\tilde{V}\left(s_{j+1}\right)=s_{j}$ for $j \geq 1$, and that $\tilde{V}\left(s_{0}+s_{1}\right)=s_{0}$. It follows that

$$
(I-\tilde{V})\left(\sum_{j=0}^{n+1} s_{j}\right)=s_{n+1} \stackrel{w}{\longrightarrow} 0
$$

in $J\left(h_{j}\right)$ as $n \rightarrow \infty$. Indeed, if $\left(s_{n+1}\right)$ is not weakly null in $J\left(h_{j}\right)$, then it would contain a subsequence equivalent to the unit vector basis of $\ell^{1}$ by [LT, 1.c.9] (recall that skipped subsequences of $\left(s_{n+1}\right)$ are unconditional, see BHO, Prop. 2.1.(2)]). This would contradict the quasi-reflexivity of $J\left(h_{j}\right)$. The proof of Theorem 4.4 is complete.

Remark 4.6. We did not consider the problem of whether the dual $J\left(h_{j}\right)^{*}$ always has the W.A.P. in the setting of Theorem 4.4.

\section{Permanence Properties AND Further Positive Results}

In this section we state some simple permanence properties for weakly compact approximation, which imply that certain vector-valued sequence spaces such as $\ell^{p}\left(\ell^{1}\right), \ell^{p}(J)$ and $\ell^{1}\left(\ell^{p}\right)$ have the W.A.P. for $1<p<\infty$. These facts will be needed in section 6 .

We first recall the following "dual" version of W.A.P., which has some applications of its own; see [T1, [T2]. The Banach space $E$ is said to have the inner weakly compact approximation property (inner W.A.P. for short) if there is a constant $C<\infty$ so that

$$
\inf \{\|U-U V\|: V \in W(E),\|V\| \leq C\}=0
$$

for any weakly compact operator $U \in W(E, Z)$, where $Z$ is an arbitrary Banach space. The inner W.A.P. is suggested by an analogous operator reformulation of the W.A.P.: $E$ has the W.A.P. if and only if there is $C<\infty$ so that

$$
\inf \{\|U-V U\|: V \in W(E),\|V\| \leq C\}=0
$$

for any weakly compact operator $U \in W(Z, E)$, where $Z$ is an arbitrary Banach space. We recall that the duality between the W.A.P. and the inner W.A.P. is incomplete.

Examples 5.1. If $E$ has the inner W.A.P., then $E^{*}$ has the W.A.P.; see [T1, 3.4]. The converse does not always hold: the Johnson-Lindenstrauss space $J L$ fails to have the inner W.A.P., but $J L^{*}$ has the W.A.P.; see [T2, 1.4]. Moreover, the fact that $E$ has the W.A.P. does not in general imply that $E^{*}$ has the inner W.A.P. (indeed, $\ell^{1}$ has the W.A.P., but $\ell^{\infty}$ does not have the inner W.A.P.; see [T1, 3.5.(ii)] or Proposition 6.10 below). Note also that $c_{0}$ has the inner W.A.P. by [T1, 3.5.(ii)], but $c_{0}$ does not have the W.A.P. 
It is a simple fact that the (inner) W.A.P. is preserved by complementation. We omit the easy arguments.

Lemma 5.2. Suppose that $M \subset E$ is a complemented subspace, and let $P$ be a projection of $E$ onto $M$.

(i) If $E$ has the W.A.P. with constant $C$, then $M$ has the W.A.P. with constant $\|P\| C$.

(ii) If $E$ has the inner W.A.P. with constant $C$, then $M$ has the inner W.A.P. with constant $\|P\| C$.

Let $R$ be a Banach space having a normalized 1-unconditional Schauder basis $\left(r_{j}\right)$ and suppose that $\left(E_{j}\right)$ is a sequence of Banach spaces. The vector-valued sequence spaces (or the $R$-direct sums)

$$
R\left(E_{j}\right)=\left\{x=\left(x_{j}\right): x_{j} \in E_{j} \text { for } j \in \mathbf{N},\|x\| \equiv\left\|\sum_{j=1}^{\infty}\right\| x_{j}\left\|\cdot r_{j}\right\|_{R}<\infty\right\}
$$

provide a suitable setting for our permanence results. Special cases include the familiar direct sums $\ell^{p}\left(E_{j}\right)(1 \leq p<\infty)$ and $c_{0}\left(E_{j}\right)$. Let $J_{k}: E_{k} \rightarrow R\left(E_{j}\right)$ denote the inclusion map and let $P_{k}$ denote the natural norm-1 projection of $R\left(E_{j}\right)$ onto $E_{k}$ for $k \in \mathbf{N}$.

Proposition 5.3. Let $\left(E_{j}\right)$ be a sequence of Banach spaces and suppose that $R$ is a reflexive Banach space having a normalized 1-unconditional Schauder basis $\left(r_{j}\right)$. Then

(i) $R\left(E_{j}\right)$ has the W.A.P. if and only if $E_{j}$ has the W.A.P. with a uniform constant for all $j$.

(ii) $R\left(E_{j}\right)$ has the inner W.A.P. if and only if $E_{j}$ has the inner W.A.P. with a uniform constant for all $j$.

(iii) $\ell^{1}\left(E_{j}\right)$ has the W.A.P. if and only if $E_{j}$ has the W.A.P. with a uniform constant for all $j$.

(iv) $c_{0}\left(E_{j}\right)$ has the inner W.A.P. if and only if $E_{j}$ has the inner W.A.P. with a uniform constant for all $j$.

Proof. (i) We put $X=R\left(E_{j}\right)$ for simplicity. If $X$ has the W.A.P. with constant $C$, then the 1-complemented subspace $E_{k} \subset X$ has the W.A.P. with the same constant $C$ for $k \in \mathbf{N}$ by Lemma $5.2(\mathrm{i})$. (The implication " $\Rightarrow$ " is checked similarly for parts (ii), (iii) and (iv).)

Conversely, assume that $E_{j}$ has the W.A.P. with a uniform constant $C$ for all $j$. Suppose that $D \subset X$ is a weakly compact subset and $\varepsilon>0$. Since $P_{k} D \subset E_{k}$ is weakly compact for $k \in \mathbf{N}$, there is by assumption $V_{k} \in W\left(E_{k}\right)$ so that

$$
\sup _{y \in P_{k} D}\left\|y-V_{k} y\right\|<\frac{\varepsilon}{2^{k}} \quad \text { and } \quad\left\|V_{k}\right\| \leq C .
$$

Define $V: X \rightarrow X$ by $V x=\left(V_{k} x_{k}\right)$ for $x=\left(x_{k}\right) \in X$. Clearly

$$
\|V x\|=\left\|\sum_{k=1}^{\infty}\right\| V_{k} x_{k}\left\|\cdot r_{k}\right\|_{R} \leq C\left\|\sum_{k=1}^{\infty}\right\| x_{k}\left\|\cdot r_{k}\right\|_{R}=C\|x\|
$$

for $x=\left(x_{k}\right) \in X$ by the 1-unconditionality of $\left(r_{k}\right)$, so that $V \in L(X)$ and $\|V\| \leq C$.

Let $\left(x^{(m)}\right) \subset X$ be a bounded sequence, where $x^{(m)}=\left(x_{j}^{(m)}\right)_{j \in \mathbf{N}} \in X$ for $m \in \mathbf{N}$. Then $x^{(m)} \stackrel{w}{\longrightarrow} x=\left(x_{j}\right)$ in $X$ as $m \rightarrow \infty$ if and only if $x_{j}^{(m)} \stackrel{w}{\longrightarrow} x_{j}$ as $m \rightarrow \infty$ in $E_{j}$ 
for $j \in \mathbf{N}$ (cf. [L, 6.1] for the special case $\ell^{p}(E)$ ). The Eberlein-Smulian theorem implies then that a bounded set $A \subset X$ is relatively weakly compact if and only if $P_{j} A$ is relatively weakly compact in $E_{j}$ for $j \in \mathbf{N}$. By applying this fact to $V B_{X}$ we get that $V \in W(X)$, since $P_{j}\left(V B_{X}\right)=V_{j} B_{E_{j}}$ is relatively weakly compact for each $j$. Finally, for $x=\left(x_{k}\right) \in D$ we have

$$
\|x-V x\|=\left\|\sum_{k=1}^{\infty}\right\| x_{k}-V_{k} x_{k}\left\|\cdot r_{k}\right\|_{R} \leq \sum_{k=1}^{\infty} \frac{\varepsilon}{2^{k}}=\varepsilon .
$$

(ii) Suppose $E_{j}$ has the inner W.A.P. with a uniform constant $C$ for all $j$. Let $U \in W(X, Z)$ and $\varepsilon>0$ be given, where $X=R\left(E_{j}\right)$ and $Z$ is some Banach space. Put $U_{k}=U J_{k} \in W\left(E_{k}, Z\right)$ for $k \in \mathbf{N}$. By assumption there is $V_{k} \in W\left(E_{k}\right)$ satisfying

$$
\left\|U_{k}-U_{k} V_{k}\right\|<\frac{\varepsilon}{2^{k}} \quad \text { and } \quad\left\|V_{k}\right\| \leq C
$$

for $k \in \mathbf{N}$. Define $V \in L(X)$ by $V x=\left(V_{k} x_{k}\right)$ for $x=\left(x_{k}\right) \in X$. One verifies as in part (i) that $\|V\| \leq C$ and $V \in W(X)$.

We next estimate $\|U-U V\|=\left\|U^{*}-V^{*} U^{*}\right\|$. Observe that $X^{*}=R^{*}\left(E_{j}^{*}\right)$, where the biorthogonal sequence $\left(r_{j}^{*}\right)$ to $\left(r_{j}\right)$ is a 1-unconditional Schauder basis for $R^{*}$. Here $U^{*}=\left(U_{j}^{*}\right): Z^{*} \rightarrow R^{*}\left(E_{j}^{*}\right)$, and we get for $z^{*} \in B_{Z^{*}}$ that

$$
\left\|U^{*} z^{*}-V^{*} U^{*} z^{*}\right\|=\left\|\sum_{k=1}^{\infty}\right\| U_{k}^{*} z^{*}-V_{k}^{*} U_{k}^{*} z^{*}\left\|\cdot r_{k}^{*}\right\|_{R^{*}} \leq \varepsilon .
$$

Hence $X=R\left(E_{j}\right)$ has the inner W.A.P.

(iii) Let $D \subset \ell^{1}\left(E_{j}\right)$ be a weakly compact set and $\varepsilon>0$. Using Lemma 5.4 below we fix $n \in \mathbf{N}$ so that

$$
\sup _{x=\left(x_{j}\right) \in D}\left(\sum_{j=n+1}^{\infty}\left\|x_{j}\right\|\right)<\frac{\varepsilon}{2} .
$$

The assumption gives operators $V_{j} \in W\left(E_{j}\right)$ for $j=1, \ldots, n$ satisfying $\left\|V_{j}\right\| \leq$ $C$ and $\left\|x_{j}-V_{j} x_{j}\right\|<\frac{\varepsilon}{2^{j+1}}$ for $x=\left(x_{k}\right) \in D$. Define $V \in L(X)$ by $V\left(x_{k}\right)=$ $\left(V_{1} x_{1}, \ldots, V_{n} x_{n}, 0,0, \ldots\right)$ for $\left(x_{k}\right) \in X$. Clearly $\|V\| \leq C$ and $V \in W(X)$. For $x=\left(x_{j}\right) \in D$ we get that

$$
\|x-V x\| \leq \sum_{j=1}^{n}\left\|x_{j}-V_{j} x_{j}\right\|+\sum_{j=n+1}^{\infty}\left\|x_{j}\right\| \leq \varepsilon .
$$

(iv) Put $X=c_{0}\left(E_{j}\right)$, and suppose that $U=\left(U_{k}\right) \in W(X, Z)$ and $\varepsilon>0$. Here $U_{k}=U J_{k}$ for $k \in \mathbf{N}$ and $U^{*}=\left(U_{k}^{*}\right)$ is a weakly compact operator. By applying Lemma 5.4 (see below) to the relatively weakly compact set $U^{*}\left(B_{Z^{*}}\right) \subset X^{*}=$ $\ell^{1}\left(E_{j}^{*}\right)$ there is $n \in \mathbf{N}$ so that

$$
\sup _{z^{*} \in B_{Z^{*}}}\left(\sum_{j=n+1}^{\infty}\left\|U_{j}^{*} z^{*}\right\|\right)<\frac{\varepsilon}{2} .
$$

Fix $V_{j} \in W\left(E_{j}\right)$ so that $\left\|U_{j}-U_{j} V_{j}\right\|<\frac{\varepsilon}{2^{j+1}}$ and $\left\|V_{j}\right\| \leq C$ for $j=1, \ldots, n$. Define again $V \in W(X)$ by $V\left(x_{k}\right)=\left(V_{1} x_{1}, \ldots, V_{n} x_{n}, 0,0, \ldots\right)$ for $\left(x_{k}\right) \in X$, so that $\|V\| \leq C$. For $z^{*} \in B_{Z^{*}}$ we get that

$\left\|U^{*} z^{*}-V^{*} U^{*} z^{*}\right\| \leq \sum_{j=1}^{n}\left\|U_{j}^{*} z^{*}-V_{j}^{*} U_{j}^{*} z^{*}\right\|+\sum_{j=n+1}^{\infty}\left\|U_{j}^{*} z^{*}\right\| \leq \sum_{j=1}^{n}\left\|U_{j}-U_{j} V_{j}\right\|+\frac{\varepsilon}{2} \leq \varepsilon$.

Thus $\|U-U V\| \leq \varepsilon$, so that $c_{0}\left(E_{j}\right)$ has the inner W.A.P. 
The following auxiliary fact was used in the proof of parts (iii) and (iv) of Proposition 5.3. We sketch the argument of this well-known result for completeness.

Lemma 5.4. Let $\left(E_{j}\right)$ be a sequence of Banach spaces and suppose that $D \subset \ell^{1}\left(E_{j}\right)$ is a weakly compact set. Then for any $\delta>0$ there is $n=n(D, \delta) \in \mathbf{N}$ so that

$$
\sup _{y=\left(y_{j}\right) \in D}\left(\sum_{j=n}^{\infty}\left\|y_{j}\right\|\right) \leq \varepsilon .
$$

Proof. Suppose to the contrary that (5.2) does not hold: there is a $\delta>0$ so that for each $n \in \mathbf{N}$ there is some $y=\left(y_{j}\right) \in D$ satisfying $\sum_{j=n}^{\infty}\left\|y_{j}\right\|>\delta$. Hence there are sequences $\left(p_{n}\right) \subset \mathbf{N}$ and $\left(y^{(n)}\right) \subset D$ so that $\sum_{j=p_{n}+1}^{p_{n+1}}\left\|y_{j}^{(n)}\right\|>\delta$ for $n \in \mathbf{N}$. Here $y^{(n)}=\left(y_{j}^{(n)}\right)_{j \in \mathbf{N}} \in \ell^{1}\left(E_{j}\right)$ for $n \in \mathbf{N}$. It is then easy to verify that a subsequence of $\left(y^{(n)}\right)$ is equivalent to the unit vector basis of $\ell^{1}$ (this will contradict the weak compactness of $D$ ).

The following novel examples of concrete spaces that have the W.A.P. (or the inner W.A.P.) are immediate from Proposition 5.3.

Corollary 5.5. Let $1<p<\infty$. Then

(i) $\ell^{p}\left(\ell^{1}\right)$ and $\ell^{1}\left(\ell^{p}\right)$ have the W.A.P.,

(ii) $\ell^{p}\left(c_{0}\right)$ and $c_{0}\left(\ell^{p}\right)$ have the inner W.A.P.,

(iii) $\ell^{p}(J)$ and $\ell^{p}\left(J^{*}\right)$ have the W.A.P.

Proof. Recall that $\ell^{1}$ has the W.A.P., and that $c_{0}$ has the inner W.A.P. (see T1. 3.5.(ii)]). Part (iii) follows from Theorems 2.2, 3.3 and Proposition 5.3(i).

Remark 5.6. The fact that $\ell^{2}(J)$ has the W.A.P. sheds some further light on a result of [GST]. Let $E$ be a Banach space and define the "residual" operator $R(S) \in L\left(E^{* *} / E\right)$ by

$$
R(S)\left(x^{* *}+E\right)=S^{* *} x^{* *}+E \quad \text { for } x^{* *} \in E^{* *}, S \in L(E) .
$$

It is known (cf. [GST, 1.4] that $\|R(S)\| \leq \omega(S) \leq\|S\|_{w}$ for $S \in L(E)$, where $\|S\|_{w} \equiv \operatorname{dist}(S, W(E))$ and $\omega(\cdot)$ is the measure of weak non-compactness (cf. the Introduction).

According to [GST 2.6] there is a sequence $\left(S_{n}\right) \subset L\left(\ell^{2}(J)\right)$ so that $\left\|S_{n}\right\|_{w}=1$ for all $n$, but $\left\|R\left(S_{n}\right)\right\| \rightarrow 0$ as $n \rightarrow \infty$. The precise relation between $\omega$ and $\|\cdot\|_{w}$ on $L\left(\ell^{2}(J)\right)$ was not resolved in [GST]. Now an inspection of the arguments of Proposition 5.3(i) and [AT, Thm. 1] reveals that in fact

$$
\omega(S) \leq\|S\|_{w} \leq 2 \omega(S), \quad S \in L\left(\ell^{2}(J)\right) .
$$

Another natural permanence problem, which we did not pursue here, concerns the W.A.P. for the Bochner spaces $L^{p}(E)$.

Problem 5.7. Does $L^{p}(E)=L^{p}([0,1] ; E)$ have the W.A.P. (resp., the inner W.A.P.) whenever $E$ has the W.A.P. (resp., the inner W.A.P.) and $1<p<\infty$ ? (The cases $p=1$ and $p=\infty$ are excluded by known facts; cf. Proposition 6.10). 


\section{James' tree space JT does not have the W.A.P. AND RELATED EXAMPLES}

This section provides concrete answers to various natural questions about the class of spaces having the W.A.P. We first recall a couple of notions. Let $1 \leq p<\infty$ be fixed. The Banach space $E$ is $\ell^{p}$-saturated if every infinite-dimensional subspace $M \subset E$ contains an isomorphic copy of $\ell^{p}$. The space $E$ is somewhat reflexive if every infinite-dimensional subspace $M \subset E$ contains a reflexive infinite-dimensional subspace. (Here "subspace" always means a closed linear subspace.)

The quasi-reflexive H.I. space $E$ from [ArT, Prop. 14.10] (cf. Example 4.1) that fails the W.A.P. yields a striking counterexample to the following question stated in $\mathrm{AT}$.

Question 6.1 ([AT p. 370]). Suppose that the quotient $E^{* *} / E$ is reflexive. Does $E$ have the W.A.P.?

Since $\ell^{2}(J)$ has the W.A.P. by Corollary 5.5(iii), and $\ell^{2}(J)^{* *} / \ell^{2}(J)$ $=\ell^{2}\left(J^{* *} / J\right)=\ell^{2}$, there are spaces $E$ with the W.A.P. and $E^{* *} / E$ reflexive and infinite-dimensional. Theorem 6.5 below yields a concrete space $Y$ without the W.A.P. for which $Y^{* *} / Y=\ell^{2}$.

Our next question addresses another potential extension of the fact that reflexive spaces have the W.A.P.

Question 6.2. Suppose that $E$ is a somewhat reflexive space that has the bounded approximation property (B.A.P.). Does $E$ have the W.A.P.?

The answer to Question 6.2 can be deduced from known results (Theorem 6.5 below contains a different, $\ell^{2}$-saturated example).

Example 6.3. Let $E$ be the separable, somewhat reflexive $\mathcal{L}^{\infty}$-space constructed by Bourgain and Delbaen; see [B] Ch. III] for a description. Then $E$ has the B.A.P., but $E$ does not have the W.A.P. (see Proposition 6.10 below or [AT, Cor. 3]).

James' tree space $J T$ was introduced by James [J2] as a useful variation of the ideas underlying $J$, and its properties were further analyzed e.g. by Lindenstrauss and Stegall [LS]. There is a systematic exposition of the properties of JT in FG. chapter 3]. The fact that $J$ has the W.A.P. (section 2) suggests the following problem.

Question 6.4. Does JT have the W.A.P.?

The main result of this section (Theorem 6.5) establishes that JT does not have the W.A.P., where $J T^{* *} / J T$ is a non-separable Hilbert space. We recall the definition of $J T$ and fix some relevant notation. Let

$$
\mathcal{T}=\bigcup_{n=0}^{\infty}\{0,1\}^{n}
$$

be the infinite binary tree equipped with the natural partial order. The nodes $\alpha \in \mathcal{T}$ satisfy $\alpha=\emptyset$ or $\alpha=\left(\alpha_{1}, \ldots, \alpha_{n}\right)$ for some $n \in \mathbf{N}$, where $\alpha_{j}=0$ or $\alpha_{j}=1$ for $j \in\{1, \ldots, n\}$. The length $|\alpha|$ of $\alpha=\left(\alpha_{1}, \ldots, \alpha_{n}\right) \in \mathcal{T}$ is $n$. Given $\alpha \in \mathcal{T}$ let $f_{\alpha}: \mathcal{T} \rightarrow \mathbf{R}$ be defined by $f_{\alpha}(\alpha)=1$ and $f_{\alpha}(\beta)=0$ for $\beta \neq \alpha$. James' tree space 
$J T$ consists of the functions $\sum_{\alpha} a_{\alpha} f_{\alpha}: \mathcal{T} \rightarrow \mathbf{R}$ for which the norm

$$
\left\|\sum_{\alpha} a_{\alpha} f_{\alpha}\right\|=\sup _{k ; S_{1}, \ldots, S_{k}}\left(\sum_{j=1}^{k} S_{j}^{*}\left(\sum_{\alpha} a_{\alpha} f_{\alpha}\right)^{2}\right)^{1 / 2}<\infty
$$

where the supremum is taken over disjoint segments $S_{1}, \ldots, S_{k}$ of $\mathcal{T}$ and $k \in \mathbf{N}$. A segment $S \subset \mathcal{T}$ has the form $S=\{\gamma \in \mathcal{T}: \alpha \leq \gamma \leq \beta\}$ for given $\alpha, \beta \in \mathcal{T}$ with $\alpha \leq \beta$, and $S^{*}\left(\sum_{\alpha} a_{\alpha} f_{\alpha}\right)=\sum_{\alpha \in S} a_{\alpha}$ for $\sum_{\alpha} a_{\alpha} f_{\alpha} \in J T$. It is known that $\left(f_{\alpha}\right)_{\alpha \in \mathcal{T}}$ is a monotone boundedly complete basis for JT (ordered by increasing length of the nodes and from "left to right").

A branch $B \subset \mathcal{T}$ is a maximal infinite order interval starting at $\emptyset$. A branch $B$ determines the norm- 1 functional $S_{B}^{*} \in J T^{*}$ defined by $S_{B}^{*}\left(\sum_{\alpha} a_{\alpha} f_{\alpha}\right)=\sum_{\alpha \in B} a_{\alpha} f_{\alpha}$. Let $\Gamma$ be the uncountable collection of all branches of $\mathcal{T}$. Then $J T^{* *} / J T=\ell^{2}(\Gamma)$ isometrically, and

$$
J T^{*}=\left[\left\{f_{\alpha}^{*}: \alpha \in \mathcal{T}\right\} \cup\left\{S_{B}^{*}: B \in \Gamma\right] ;\right.
$$

see [LS, Thm. 1] or [FG, 3.c.3]. Here $\left(f_{\alpha}^{*}\right)_{\alpha \in \mathcal{T}}$ is the biorthogonal sequence to $\left(f_{\alpha}\right)_{\alpha \in \mathcal{T}}$ in $J T^{*}$. Recall further that $J T$ is $\ell^{2}$-saturated; see [J2, Thm.] or [FG. 3.a.8].

The following special notation will be convenient. If $\alpha=\left(\alpha_{1}, \ldots, \alpha_{n}\right) \in \mathcal{T}$, then $\alpha 0=\left(\alpha_{1}, \ldots, \alpha_{n}, 0\right)$ is the left successor (or left concatenation) and $\alpha 1=$ $\left(\alpha_{1}, \ldots, \alpha_{n}, 1\right)$ the right successor of $\alpha$. For any $n \in \mathbf{N}$ we put

$$
\mathcal{T}_{n}=\{\alpha \in \mathcal{T}: \text { there are at most } n 1 \text { 's in } \alpha\} .
$$

Thus $\alpha \in \mathcal{T}_{n}$ if the node $\alpha$ contains at most $n$ "right turns". Put $X_{n}=\left[f_{\alpha}: \alpha \in \mathcal{T}_{n}\right]$ for $n \in \mathbf{N}$. Note that $X_{n} \subset J T$ is a 1-complemented subspace, where the restriction $x \mapsto x_{\mid \mathcal{T}_{n}}$ defines the natural projection onto $X_{n}$. Indeed, if $S_{1}, \ldots, S_{k} \subset \mathcal{T}$ are disjoint segments, then $S_{j} \cap \mathcal{T}_{n}$ are disjoint segments (possibly empty) in $\mathcal{T}_{n}$ for $j=1, \ldots, k$.

We are ready for our main results about $J T$. Below parts (i) and (ii) together imply that $X_{n}$ has the W.A.P. for all $n \in \mathbf{N}$, but where the smallest constant $C$ in (1.1) is proportional to $\sqrt{n}$. Parts (iii) and (iv) are only based on (ii), but (i) will become useful later (see Example 6.8 and Remark 6.9). The space $Y$ in part (iv) is an example, where $Y$ fails to have the W.A.P. and the reflexive quotient $Y^{* *} / Y$ is much "smaller" than $J T^{* *} / J T$. The quasi-reflexive space from ArT, Prop. 14.10] yields an optimal negative answer to Question 6.1 in terms of minimizing $\operatorname{dim}\left(Y^{* *} / Y\right)$, but our examples are easier. They are further witnesses that the quotient $E^{* *} / E$ alone does not decide the W.A.P. of $E$.

Theorem 6.5. Let $X_{n}=\left[f_{\alpha}: \alpha \in \mathcal{T}_{n}\right] \subset J T$ be as above. Then the following properties hold:

(i) $X_{n}$ has the W.A.P. with constant (at most) $3 \sqrt{n}$ for $n \in \mathbf{N}$.

(ii) There is a uniform constant $C>0$ with the following property: for each $n \in \mathbf{N}$ there is a weakly compact set $D_{n} \subset X_{n}$ so that if

$$
\sup _{x \in D_{n}}\|x-V x\|<\frac{1}{10} \quad \text { and } V \in W\left(X_{n}\right),
$$

then $\|V\| \geq C \sqrt{n}$.

(iii) JT does not have the W.A.P. 
(iv) If $Y=\ell^{2}\left(X_{n}\right)$, then $Y^{* *} / Y=\ell^{2}$ isometrically and $Y$ does not have the W.A.P.

Proof. We say that $B \subset \mathcal{T}$ is a branch starting at the node $\alpha \in \mathcal{T}$ if $B$ is a maximal infinite linearly ordered set so that $\gamma \geq \alpha$ for all $\gamma \in B$. It is convenient to fix, for each $n \in \mathbf{N}$, a partition

$$
\mathcal{T}_{n}=\bigcup_{j=1}^{\infty} B_{j}^{(n)}
$$

into disjoint branches, where every $B_{j}^{(n)}=\left(\alpha^{(j, n)}, \alpha^{(j, n)} 0, \alpha^{(j, n)} 00, \ldots\right)$ is the "always left" branch starting at some node $\alpha^{(j, n)}=\left(\alpha_{1}^{(j, n)}, \ldots, \alpha_{k}^{(j, n)}\right) \in \mathcal{T}_{n}$ with $\alpha_{k}^{(j, n)}=1$. We may enumerate these branches by requiring that $B_{r+1}^{(n)}$ starts at the first node $\alpha \in \mathcal{T}_{n} \backslash \bigcup_{j=1}^{r} B_{j}^{(n)}$ (enumerated according to increasing length and from "left to right").

(i) We will apply Proposition 5.3. Fix $n \in \mathbf{N}$ and put $B_{j} \equiv B_{j}^{(n)}$ for notational simplicity as $j \in \mathbf{N}$. Note that $Y_{j} \equiv\left[f_{\alpha}: \alpha \in B_{j}\right]=J$ isometrically for $j \in \mathbf{N}$ according to (6.1) and (3.1). Let $P_{j} \in L\left(X_{n}\right)$ be the natural norm-1 projection onto $Y_{j}$ corresponding to the restriction $x \mapsto x_{\mid B_{j}}$ for $j \in \mathbf{N}$.

Define a linear map $T: X_{n} \rightarrow \ell^{2}(J)$ by $T x=\left(P_{j} x\right)$ for $x \in X_{n}$. It will be enough to show that $T$ is an isomorphism satisfying $\|T\| \cdot\left\|T^{-1}\right\| \leq \sqrt{n}$. Indeed, recall that according to Theorem 2.2 and Proposition 5.3(i) the direct sum $\ell^{2}(J)$ has the W.A.P. with constant $C \leq 3$ as defined by (1.1). It is then straightforward to check (using the isomorphism $T$ ) that $X_{n}$ has the W.A.P. with some constant $\tilde{C} \leq 3 \sqrt{n}$.

We claim that the following estimates hold, where the left-hand inequality of (6.4) states that $T$ is well defined $X_{n} \rightarrow \ell^{2}(J)$.

Claim 1. If $x \in X_{n}$, then

$$
\left(\sum_{j=1}^{\infty}\left\|P_{j} x\right\|^{2}\right)^{1 / 2} \leq\|x\| \leq \sqrt{n} \cdot\left(\sum_{j=1}^{\infty}\left\|P_{j} x\right\|^{2}\right)^{1 / 2} .
$$

Proof of Claim 1. We may write $x \in X_{n}$ coordinatewise as $x=\sum_{j=1}^{\infty} P_{j} x=$ $\sum_{j=1}^{\infty} x_{\mid B_{j}}$, since the branches $\left\{B_{j}: j \in \mathbf{N}\right\}$ form a partition of $\mathcal{T}_{n}$. The left-hand inequality in (6.4) is then obvious by selecting segments that approximately norm each $P_{j} x$ and which are wholly contained in $B_{j}$.

Suppose next that $S_{1}, \ldots, S_{m} \subset \mathcal{T}_{n}$ are disjoint segments. According to (6.1) we must show that

$$
\sum_{j=1}^{m} S_{j}^{*}(x)^{2} \leq n \cdot \sum_{r=1}^{\infty}\left\|P_{r} x\right\|^{2}
$$

for $x \in X_{n}$. Recall that the nodes $\alpha \in \mathcal{T}_{n}$ have at most $n$ right turns, so that

$$
n(j) \equiv\left|\left\{r \in \mathbf{N}: S_{j} \cap B_{r} \neq \emptyset\right\}\right| \leq n
$$

for $j=1, \ldots, m$. Write the resulting intersected segments as $S_{j, j(1)}, \ldots, S_{j, j(n)}$, where we put $S_{j, j(r)}=\emptyset$ if $n(j)<r \leq n$. We may thus write $S_{j}^{*}(x)=\sum_{r=1}^{n} S_{j, j(r)}^{*}(x)$ for $x \in X_{n}$ and $j=1, \ldots, m$ (observing the convention that $S_{j, j(r)}^{*}(x)=0$ if $\left.S_{j, j(r)}=\emptyset\right)$. Here $\left(\sum_{r=1}^{n} S_{j, j(r)}^{*}(x)\right)^{2} \leq n \cdot\left(\sum_{r=1}^{n} S_{j, j(r)}^{*}(x)^{2}\right)$ by Hölder's inequality. 
Hence we get from the above that

$$
\sum_{j=1}^{m} S_{j}^{*}(x)^{2}=\sum_{j=1}^{m}\left(\sum_{r=1}^{n} S_{j, j(r)}^{*}(x)\right)^{2} \leq n \cdot \sum_{j=1}^{m}\left(\sum_{r=1}^{n} S_{j, j(r)}^{*}(x)^{2}\right) \leq n \cdot \sum_{s=1}^{\infty}\left\|P_{s} x\right\|^{2} .
$$

For the last estimate regroup the finite sum into those of the disjoint segments $\left\{S_{j, j(r)}: j=1, \ldots, m, r=1, \ldots, n\right\}$ that lie inside any given branch $B_{s}$ for $s \in \mathbf{N}$.

(ii) Let $n \geq 6$ be fixed. For simplicity we put again $B_{j} \equiv B_{j}^{(n)}, j \in \mathbf{N}$, for the partition $\mathcal{T}_{n}=\bigcup_{j=1}^{\infty} B_{j}^{(n)}$ that was fixed at the beginning of the proof. Consider the subset

$$
D_{n}=\bigcup_{\alpha \in \mathcal{T}_{n}}\left\{f_{\alpha}-f_{\alpha 0}, f_{\alpha 0}-f_{\alpha 00}, \ldots\right\}=\bigcup_{j=1}^{\infty}\left\{f_{\alpha}-f_{\alpha 0}: \alpha \in B_{j}\right\} \subset X_{n} .
$$

Here the sequences $\left(f_{\alpha}-f_{\alpha 0}\right)_{\alpha \in B_{j}}$ are formed by the consecutive differences along the "always left" branches $B_{j}$ in $\mathcal{T}_{n}$ for $j \in \mathbf{N}$.

Claim 2. The set $D_{n} \cup\{0\}$ is weakly compact in $X_{n}$.

Proof of Claim 2. We will verify that any sequence $\left(x_{m}\right)=\left(f_{\alpha_{m}}-f_{\alpha_{m} 0}\right)$ of distinct points from $D_{n}$ contains a weak-null subsequence $\left(x_{m_{k}}\right)$ in $J T$.

There is no loss of generality to assume, by applying Ramsey's classical theorem and passing to a subsequence of $\left(x_{m}\right)$, that either

$$
\begin{aligned}
& \text { all the nodes } \alpha_{m} \text { lie on a single branch } B \text { of } \mathcal{T} \text {, or } \\
& \text { all the nodes } \alpha_{m} \text { are pairwise incomparable. }
\end{aligned}
$$

If (6.5) holds, then $\left(x_{m}\right)$ is equivalent to a subsequence of the shrinking basis $\left(e_{n}\right)$ of $J$ given by (2.1), and hence it is weakly null (cf. [FG, 2.c.10]). If (6.6) holds, then $\left(x_{m}\right)$ is equivalent to the unit vector basis of $\ell^{2}$, and hence it is again weakly null. Thus Claim 2 holds.

Suppose next that $V \in L\left(X_{n}\right)$ is a weakly compact operator satisfying (6.3) for the weakly compact set $D_{n} \cup\{0\}$. For simplicity, let $\left(f_{m}\right)$ stand for the node basis of a given branch $B_{r}$ of the partition of $\mathcal{T}_{n}$. We make a preliminary observation.

Fact. Given $\delta>0$ there is a sequence of disjointly supported convex blocks $\left(g_{j}\right)$ of $\left(f_{m}\right)$ so that

$$
\left\|V g_{j}-V g_{i}\right\|<\delta, \quad i \neq j .
$$

Here $\left\|g_{j}\right\|=1$ for $j \in \mathbf{N}$ in view of (6.1).

Indeed, the weak compactness of $V$ yields a subsequence $\left(f_{m_{i}}\right)$ so that $V f_{m_{i}} \stackrel{w}{\longrightarrow}$ $x \in J T$ as $i \rightarrow \infty$. Then Mazur's theorem gives a sequence of disjointly supported convex blocks $\left(g_{j}\right)$ of $\left(f_{m_{i}}\right)$ so that $\left\|V g_{j}-V g_{i}\right\|<\delta$ whenever $i \neq j$.

Let $[t]$ denote the integer part of $t>0$. We successively apply the preceding Fact to $[n / 2]$ "adjacent" branches in $\mathcal{T}_{n}$, in the manner described below, to get the element

$$
x_{n}=\sum_{j=1}^{[n / 2]}\left(g_{2 j}-g_{2 j-1}\right)+\sum_{j=1}^{[n / 2]}\left(f_{\alpha_{j}}-f_{\alpha_{j} 0}\right) \in X_{n} .
$$


The differences $g_{2 j}-g_{2 j-1}$ and $f_{\alpha_{j}}-f_{\alpha_{j} 0}$ are successively chosen as follows for $j=1, \ldots,[n / 2]$ :

$g_{1}$ and $g_{2}$ are normalized convex blocks on the node basis determined by the "always left" branch $B_{1}$, their supports satisfy max $\operatorname{supp}\left(g_{1}\right)<\gamma_{1}<\min \operatorname{supp}\left(g_{2}\right)$ for some node $\gamma_{1} \in$ $B_{1}$, and $\left\|V g_{2}-V g_{1}\right\|<\frac{1}{10}$. (Here the support of the convex combinations is with respect to the node basis.)

$$
\alpha_{1}=\gamma_{1} 1 \text { (the right successor of } \gamma_{1} \text { ). }
$$

To continue, repeat the above procedure by applying (6.7) to the "always left" branch in $\mathcal{T}_{n}$ starting from the node $\alpha_{1} 1=\gamma_{1} 11$ (the right successor of $\alpha_{1}$ ). A picture will be helpful at this stage. This construction can be performed $[n / 2]$ times, since $\mathcal{T}_{n}$ allows at most $n$ right turns.

Claim 3.

$$
\left\|x_{n}\right\| \leq \sqrt{6 n} \text { and }\left\|V x_{n}\right\| \geq \frac{n}{3}
$$

Clearly (6.11) yields that $\|V\| \geq \frac{1}{\sqrt{6 n}} \cdot \frac{n}{3}=\frac{1}{3 \sqrt{6}} \cdot \sqrt{n}$, which gives (ii) with $C=\frac{1}{3 \sqrt{6}}$.

Proof of Claim 3. Let $S_{1}, \ldots, S_{m} \subset \mathcal{T}_{n}$ be given disjoint segments. We have to verify that

$$
\left(\sum_{j=1}^{m} S_{j}^{*}\left(x_{n}\right)^{2}\right)^{1 / 2} \leq \sqrt{6 n}
$$

Note that $x_{n}$ is a sum of $4\left[\frac{n}{2}\right]$ normalized blocks in $J T$, namely the convex blocks $g_{i}$ for $i=1, \ldots, 2\left[\frac{n}{2}\right]$, and $f_{\alpha_{j}}$ and $f_{\alpha_{j} 0}$ for $j=1, \ldots,\left[\frac{n}{2}\right]$. From the iterative construction of $x_{n}$ it follows that for each segment $S_{j}$ there are at most 3 nonempty disjoint segments $S_{j, 1}, S_{j, 2}, S_{j, 3} \subset S_{j}$ so that

$$
S_{j}^{*}\left(x_{n}\right)=\sum_{i=1}^{3} S_{j, i}^{*}\left(x_{n}\right),
$$

each $S_{j, i}$ is contained in the smallest segment containing one of the blocks forming $x_{n}$.

Let $T_{s}$ be the smallest segment in $\mathcal{T}_{n}$ containing $\operatorname{supp}\left(g_{s}\right)$ for $s=1, \ldots, 2\left[\frac{n}{2}\right]$. Note that $\sum_{r=1}^{p} U_{r}^{*}\left(x_{n}\right)^{2} \leq\left\|g_{s}\right\|^{2}=1$ for each $s$, whenever $U_{1}, \ldots, U_{p}$ are disjoint segments contained in $T_{s}$. Hence it follows from Hölder's inequality and (6.12), (6.13) that

$$
\left(\sum_{j=1}^{m} S_{j}^{*}\left(x_{n}\right)^{2}\right)^{1 / 2} \leq \sqrt{3} \sqrt{4\left[\frac{n}{2}\right]} \leq \sqrt{6 n} .
$$

This yields the first estimate in (6.11).

Note for the second estimate in (6.11) that according to assumption (6.3) one has

$$
V\left(f_{\alpha_{j}}-f_{\alpha_{j} 0}\right)=f_{\alpha_{j}}-f_{\alpha_{j} 0}+z_{j}
$$

where $\left\|z_{j}\right\|<\frac{1}{10}$ for $j=1, \ldots,[n / 2]$, since $f_{\alpha_{j}}-f_{\alpha_{j} 0} \in D_{n}$. Let $S \subset \mathcal{T}$ be a segment so that $\alpha_{j} \in S$, but its left successor $\alpha_{j} 0 \notin S$ for $j=1, \ldots,[n / 2]$. Then we get that

$$
\left\|\sum_{j=1}^{[n / 2]}\left(f_{\alpha_{j}}-f_{\alpha_{j} 0}\right)\right\| \geq\left|S^{*}\left(\sum_{j=1}^{[n / 2]}\left(f_{\alpha_{j}}-f_{\alpha_{j} 0}\right)\right)\right|=\left[\frac{n}{2}\right] .
$$


Since $\left\|V\left(g_{2 j}-g_{2 j-1}\right)\right\|<\frac{1}{10}$ for $j=1, \ldots,[n / 2]$ by construction, we obtain that

$$
\begin{aligned}
\left\|V x_{n}\right\| & =\left\|\sum_{j=1}^{[n / 2]} V\left(g_{2 j}-g_{2 j-1}\right)+\sum_{j=1}^{[n / 2]} V\left(f_{\alpha_{j}}-f_{\alpha_{j} 0}\right)\right\| \\
& \geq\left\|\sum_{j=1}^{[n / 2]}\left(f_{\alpha_{j}}-f_{\alpha_{j} 0}\right)\right\|-\sum_{j=1}^{[n / 2]}\left\|z_{j}\right\|-\sum_{j=1}^{[n / 2]}\left\|V\left(g_{2 j}-g_{2 j-1}\right)\right\| \\
& \geq\left[\frac{n}{2}\right]-\frac{n}{2}\left(\frac{1}{10}+\frac{1}{10}\right) \geq \frac{n}{3} .
\end{aligned}
$$

(iii) This fact follows from part (ii) and Lemma 5.2(i), since $D_{n} \subset X_{n} \subset J T$, where $X_{n}$ is 1-complemented in $J T$ for all $n \in \mathbf{N}$

(iv) The direct sum $Y=\ell^{2}\left(X_{n}\right)$ does not have the W.A.P. in view of Proposition 5.3(i), since according to part (ii) the spaces $X_{n}$ do not have the W.A.P. with a uniform constant. A modification of the corresponding argument for $J T$ in LS Thm. 1] (see also [FG, 3.c.3]) yields that $X_{n}^{* *} / X_{n}=\ell^{2}$ isometrically for all $n \in \mathbf{N}$. Hence $\ell^{2}\left(X_{n}\right)^{* *} / \ell^{2}\left(X_{n}\right)=\ell^{2}\left(X_{n}^{* *} / X_{n}\right)=\ell^{2}$.

Remark 6.6. Lindenstrauss and Stegall [LS defined a function space version of $J$. James' function space $J F$ does not have the W.A.P., since the separable space $J F$ contains a (complemented) copy of $c_{0}$; see [LS, p. 95].

The property defined by (1.1) should more precisely be called the bounded W.A.P. We say that $E$ has the unbounded W.A.P. if for every weakly compact set $D \subset E$ and $\varepsilon>0$ there is $V \in W(E)$ satisfying

$$
\sup _{x \in D}\|x-V x\|<\varepsilon
$$

The reason for our unorthodox terminology is that the known applications of weakly compact approximation are related to the property defined by (1.1), rather than the one by (6.14). Recall that there are spaces that have the (finite rank) approximation property A.P., but not the B.A.P.; see [LT 1.e]. This raises another problem.

Question 6.7. Is there a space $E$ that has the unbounded W.A.P., but not the W.A.P.?

It turns out that Theorem 6.5 yields concrete examples of this kind, so that the unbounded W.A.P. is a strictly weaker notion than the W.A.P.

Example 6.8. Let $X_{n}=\left[f_{\alpha}: \alpha \in \mathcal{T}_{n}\right] \subset J T$ be the spaces from Theorem 6.5 for $n \in \mathbf{N}$, and let $Z=\ell^{1}\left(X_{n}\right)$ be their direct $\ell^{1}$-sum. Then $Z$ has the unbounded W.A.P., but not the W.A.P.

Proof. Proposition 5.3(iii) yields that $Z=\ell^{1}\left(X_{n}\right)$ does not have W.A.P., since the spaces $X_{n}$ do not have the W.A.P. with a uniform constant according to Theorem 6.5(ii). On the other hand, since $X_{n}$ has the W.A.P. for all $n \in \mathbf{N}$ by Theorem 6.5(i), a simple modification of the argument for Proposition 5.3(iii) implies that $Z=\ell^{1}\left(X_{n}\right)$ does have the unbounded W.A.P. Indeed, recall that the relevant approximating operators $V \in W(Z)$ were defined by $V x=\left(V_{1} x_{1}, \ldots, V_{n} x_{n}, 0,0, \ldots\right)$, $x=\left(x_{k}\right) \in Z$, for suitably chosen $n \in \mathbf{N}$ and $V_{j} \in W\left(X_{j}\right)$ for $j=1, \ldots, n$. 
Remark 6.9. The space $J T$ does not even have the unbounded W.A.P. Indeed, let

$$
\tilde{D}=\bigcup_{n=1}^{\infty} D_{n} \cup\{0\} \subset \ell^{2}\left(X_{n}\right)
$$

be the coordinatewise union in the direct $\ell^{2}$-sum, where the weakly compact sets $D_{n} \subset X_{n}$ are those of the proof of Theorem 6.5(ii) for $n \in \mathbf{N}$. The set $\tilde{D}$ is relatively weakly compact in $\ell^{2}\left(X_{n}\right)$ (cf. the proof of Proposition 5.3(i)). Note that $\ell^{2}\left(X_{n}\right) \subset \ell^{2}(J T)$, where $\ell^{2}(J T)$ embeds as a complemented subspace of $J T$, see [FG], 3.a.17]. Fix a linear embedding $T: \ell^{2}(J T) \rightarrow J T$, and a projection $P$ of $J T$ onto $T\left(\ell^{2}\left(X_{n}\right)\right)$.

Suppose that for any $\varepsilon>0$ there is $V \in W(J T)$ satisfying $\|x-V x\|<\varepsilon$ for all $x \in T(\tilde{D})$. It is then easy to check that for every $n \in \mathbf{N}$ there is $V_{n} \in W\left(X_{n}\right)$, so that

$$
\left\|V_{n}\right\| \leq C\|V\| \quad \text { and } \sup _{z \in D_{n}}\left\|z-V_{n} z\right\|<c \cdot \varepsilon,
$$

where $C>0$ and $c>0$ are uniform constants that only depend on $\|T\|,\left\|T^{-1}\right\|$ and $\|P\|$. This contradicts Theorem 6.5(ii) with $\epsilon>0$ small enough and $n \in \mathbf{N}$ large enough.

For completeness we next state two simple conditions which guarantee that spaces with the Dunford-Pettis property fail to have the (inner) W.A.P. (see also [AT, Prop. 2] and [T1, 3.3]). Recall that a Banach space $E$ has the Dunford-Pettis property (DPP) if $\left\|V x_{n}\right\| \rightarrow 0$ as $n \rightarrow \infty$ whenever $V \in W(E, F)$ and $\left(x_{n}\right) \subset E$ is a weak-null sequence. The space $E$ has the Schur property if $\left\|x_{n}\right\| \rightarrow 0$ as $n \rightarrow \infty$ for every weak-null sequence $\left(x_{n}\right) \subset E$. The survey Di contains a lot of information about the Dunford-Pettis and the Schur properties. The known facts that $L^{1}(0,1)$ and $L^{\infty}(0,1)$ have neither the W.A.P. nor the inner W.A.P., as well as many additional examples, can be recovered from the following proposition.

Proposition 6.10. Let $E$ be a Banach space having the DPP.

(i) If $E$ has the W.A.P., then $E$ has the Schur property. In particular, if $E$ contains an infinite-dimensional reflexive subspace $M$, then $E$ fails to have the W.A.P.

(ii) If $E$ has an infinite-dimensional reflexive quotient space $E / M$, then $E$ fails to have the inner W.A.P.

Proof. (i) If $E$ does not have the Schur property, then there is a weak-null sequence $\left(x_{n}\right) \subset E$ so that $\left\|x_{n}\right\| \geq c>0$ for $n \in \mathbf{N}$. Then $\left\|V x_{n}\right\| \rightarrow 0$ as $n \rightarrow \infty$ by the Dunford-Pettis property of $E$ for any $V \in W(E)$. Hence

$$
\left\|x_{n}-V x_{n}\right\| \geq c-\left\|V x_{n}\right\| \geq \frac{c}{2}
$$

for all large enough $n \in \mathbf{N}$, so that $E$ does not have the W.A.P. In particular, if $E$ contains an infinite-dimensional reflexive subspace, then $E$ cannot have the Schur property.

(ii) Let $Q: E \rightarrow E / M$ stand for the weakly compact quotient map. Suppose that there is a sequence $\left(V_{n}\right) \subset W(E)$ so that $\left\|Q-Q V_{n}\right\| \rightarrow 0$ as $n \rightarrow \infty$. It follows that $Q V_{n}$ is a compact operator $E \rightarrow E / M$ for $n \in \mathbf{N}$, since $E$ has the DPP. Hence the quotient map $Q$ is a compact operator onto $E / M$, which is not possible. 
Note that $c_{0}$ has the property that every infinite-dimensional subspace $M \subset c_{0}$ fails to have the W.A.P. This follows from Proposition 6.10(i) and the fact that $c_{0}$ is complementedly $c_{0}$-saturated; see [LS, 2.a.2]. This fact is another point of difference between the W.A.P. and classical approximation properties.

It is clear that $E$ has the W.A.P. if $E$ has the Schur property and the B.A.P., since $W(E)=K(E)$ in this case. Any space $E$ with the Schur property is $\ell^{1}$-saturated by Rosenthal's $\ell^{1}$-theorem (see $[\mathrm{LT}, 2 . \mathrm{e} .5]$ ). This fact suggests the following question.

Question 6.11. Suppose that $E$ is an $\ell^{1}$-saturated Banach space that has the B.A.P. Does $E$ have the W.A.P.?

We answer Question 6.11 by showing that the Lorentz sequence spaces $d(w, 1)$ fail to have the W.A.P. Let $w=\left(w_{j}\right)$ be a positive non-increasing sequence satisfying

$$
w_{1}=1, \quad \lim _{j \rightarrow \infty} w_{j}=0 \quad \text { and } \sum_{j=1}^{\infty} w_{j}=\infty
$$

Recall that $d(w, 1)$ consists of the scalar sequences $x=\left(x_{j}\right)$ for which

$$
\|x\|=\sum_{j=1}^{\infty} w_{j} x_{j}^{*}<\infty
$$

where $\left(x_{j}^{*}\right)$ is the non-increasing rearrangement of $\left(\left|x_{j}\right|\right)$. The space $d(w, 1)$ is $\ell^{1}$ saturated by [LT, 4.e.3], but $d(w, 1)$ does not have the DPP, since the coordinate basis $\left(e_{n}\right)$ and its biorthogonal sequence $\left(e_{n}^{*}\right)$ in $d(w, 1)^{*}$ are weakly null. In place of Proposition 6.10 we will use the (sub)symmetry of the Schauder basis $\left(e_{n}\right)$ for $d(w, 1)$.

Let $E$ be a Banach space. Recall that a Schauder basis $\left(e_{n}\right)$ for $E$ is symmetric if $\left(e_{\pi(n)}\right)$ and $\left(e_{n}\right)$ are equivalent for all permutations $\pi$ of $\mathbf{N}$. The basis $\left(e_{n}\right)$ is subsymmetric if $\left(e_{n}\right)$ is unconditional and $\left(e_{m_{n}}\right)$ is equivalent to $\left(e_{n}\right)$ for all subsequences $m_{1}<m_{2}<\ldots$. Every symmetric basis is also subsymmetric [LT] 3.a.3]. Let $\left(x_{j}\right)$ and $\left(y_{j}\right)$ be basic sequences in $E$. Recall that $\left(x_{j}\right)$ is said to dominate $\left(y_{j}\right)$ if $\sum_{j=1}^{\infty} c_{j} y_{j}$ converges in $E$ whenever $\sum_{j=1}^{\infty} c_{j} x_{j}$ converges in $E$.

Example 6.12. $d(w, 1)$ does not have the W.A.P.

Proof. The set $D=\left\{e_{n}: n \in \mathbf{N}\right\} \cup\{0\} \subset d(w, 1)$ is weakly compact, since $\left(e_{n}\right)$ is a weak-null sequence. We will show that $D$ cannot be approximated in the sense of (1.1). Suppose for this purpose that $V \in L(d(w, 1))$ satisfies $\sup _{n \in \mathbf{N}}\left\|e_{n}-V e_{n}\right\|<$ $\frac{1}{10}$, and put $x_{n}=V e_{n}$ for $n \in \mathbf{N}$. Then the sequence $\left(x_{n}\right)$ is semi-normalized and weakly null.

Claim. $V \notin W(d(w, 1))$.

First choose a basic subsequence $\left(x_{n_{j}}\right)$ so that $\left(x_{n_{j}}\right)$ is equivalent to a block basic sequence $\left(y_{j}\right)$ of $\left(e_{n}\right)$, where $\left\|x_{n_{j}}-y_{j}\right\| \rightarrow 0$ as $j \rightarrow \infty$. Put $y_{j}=\sum_{k=p_{j}}^{q_{j}} a_{k} e_{k}$ for $j \in \mathbf{N}$, where $p_{1}<q_{1}<p_{2}<q_{2}<\ldots$ is a suitable sequence. It is obvious that $\left(e_{n_{j}}\right)$ dominates $\left(x_{n_{j}}\right)=\left(V e_{n_{j}}\right)$. We next verify that $\left(x_{n_{j}}\right)$ dominates $\left(e_{n_{j}}\right)$, so that $\left(x_{n_{j}}\right)$ and $\left(e_{n_{j}}\right)$ will be equivalent basic sequences in $d(w, 1)$.

We may assume by approximation that $n_{j} \in\left[p_{j}, q_{j}\right]$ and $a_{n_{j}}=e_{n_{j}}^{*}\left(y_{j}\right)>\frac{9}{10}$ for $j \in \mathbf{N}$. Let $c_{1}, \ldots, c_{r}$ be scalars and $r \in \mathbf{N}$. It follows from the 1-unconditionality 
of the basis $\left(e_{j}\right)$ that

$$
\left\|\sum_{j=1}^{r} c_{j} y_{j}\right\|=\left\|\sum_{j=1}^{r}\left(\sum_{k=p_{j}}^{q_{j}} c_{j} a_{k} e_{k}\right)\right\| \geq \frac{9}{10}\left\|\sum_{j=1}^{r} c_{j} e_{n_{j}}\right\|,
$$

and hence $\left(x_{n_{j}}\right)$ dominates $\left(e_{n_{j}}\right)$.

It follows that the restriction of $V$ determines a linear isomorphism $\left[e_{n_{j}}: j \in\right.$ $\mathbf{N}] \rightarrow\left[V e_{n_{j}}: j \in \mathbf{N}\right]$, since the sequences $\left(e_{n_{j}}\right)$ and $\left(V e_{n_{j}}\right)$ are equivalent. Here $\left[e_{n_{j}}: j \in \mathbf{N}\right] \approx d(w, 1)$, because $\left(e_{n}\right)$ is a (sub)symmetric basis. This implies the Claim.

Remark 6.13. The argument of Example 6.12 actually yields a more general observation, which applies e.g. to certain Orlicz sequence spaces (see Chapter 4 of [LT]:

Suppose that $E$ is a non-reflexive Banach space which has a weak-null, subsymmetric Schauder basis $\left(e_{n}\right)$. Then $E$ does not have the W.A.P.

Azimi and Hagler $[\mathrm{AH}]$ introduced a class of spaces that provides a second solution to Question 6.11 (with some additional properties). Let $w=\left(w_{j}\right)$ be a positive non-increasing sequence satisfying (6.15). The Azimi-Hagler space $X(w)$ consists of the scalar sequences $x=\left(x_{j}\right)$ for which

$$
\|x\|=\sup _{n ; F_{1}<\ldots<F_{n}} \sum_{j=1}^{n} w_{j}\left|\sum_{k \in F_{j}} x_{k}\right|<\infty .
$$

The supremum is taken over all finite intervals $F_{1}<\ldots<F_{n}$ of $\mathbf{N}$ and $n \in \mathbf{N}$. The Banach space $X(w)$ is $\ell^{1}$-saturated, but it does not have the Schur property; see [AH, Thm. 1]. One point of interest in $X(w)$ comes from the facts that the coordinate basis $\left(e_{n}\right)$ is not a subsymmetric basis for $X(w)$ (see the Remark on AH, p. 295]), and $\left(e_{n}\right)$ does not even contain any weakly convergent subsequences (see [AH, Thm. 1.(3)]). Hence the approach of Example 6.12 must be refined.

Let $P_{m, n}$ denote the natural projection of $X(w)$ onto $\left[e_{s}: m \leq s \leq n\right]$ for $m \leq n$. Thus $\left\|P_{m, n}\right\| \leq 2$.

Example 6.14. $X(w)$ does not have the W.A.P.

Proof. Put $z_{n}=e_{2 n}-e_{2 n-1}$ for $n \in \mathbf{N}$. Then $\left(z_{n}\right)$ is a weak-null sequence in $X(w)$ (see [AH, Lemma 6]), so that $\left\{z_{n}: n \in \mathbf{N}\right\} \cup\{0\}$ is a weakly compact set. Suppose that $V \in L(X(w))$ satisfies

$$
\sup _{n \in \mathbf{N}}\left\|z_{n}-V z_{n}\right\|<\frac{1}{10}
$$

and set $x_{n}=V z_{n}$ for $n \in \mathbf{N}$. Thus $\left(x_{n}\right)$ is a semi-normalized weak-null sequence.

Claim. $V \notin W(X(w))$.

By the standard gliding hump argument we may first choose a subsequence $\left(x_{n_{j}}\right)$ and natural numbers $p_{1}<q_{1}<p_{2}<q_{2}<p_{3}<\ldots$, so that

(i) $\left(x_{n_{j}}\right)$ and $\left(y_{j}\right)$ are equivalent basic sequences, where $y_{j}=P_{p_{j}, q_{j}}\left(x_{n_{j}}\right)$ for $j \in \mathbf{N}$

(ii) $y_{j}=u_{j}+a_{j} e_{2 n_{j}-1}+b_{j} e_{2 n_{j}}+v_{j}$, where $p_{j}<2 n_{j}-1<2 n_{j}<q_{j}$, and the supports satisfy $\operatorname{supp}\left(u_{j}\right) \subset\left[p_{j}, 2 n_{j}-1\right)$ and $\operatorname{supp}\left(v_{j}\right) \subset\left(2 n_{j}, q_{j}\right]$ for $j \in \mathbf{N}$.

(iii) $\left|a_{j}+1\right|<\frac{2}{10}$ and $\left|b_{j}-1\right|<\frac{2}{10}$ for $j \in \mathbf{N}$. 
Property (iii) follows from the fact that $\left\|y_{j}-z_{n_{j}}\right\|=\left\|P_{p_{j}, q_{j}}\left(x_{n_{j}}-z_{n_{j}}\right)\right\|<\frac{2}{10}$.

Clearly $\left(z_{n_{j}}\right)$ dominates $\left(x_{n_{j}}\right)=\left(V z_{n_{j}}\right)$. By property (i) it suffices to verify that there is $c>0$ so that

$$
\left\|\sum_{j} c_{j} y_{j}\right\| \geq c \cdot\left\|\sum_{j} c_{j} z_{n_{j}}\right\|
$$

for all scalars $c_{1}, c_{2}, \ldots$ Indeed, in that event the basic sequences $\left(x_{n_{j}}\right)$ and $\left(z_{n_{j}}\right)$ are equivalent, and the fact that $X(w)$ is $\ell^{1}$-saturated [T1, Thm. 1] will imply that $V$ fixes some $\ell^{1}$-copy contained in $\left[z_{n_{j}}: j \in \mathbf{N}\right]$.

It is enough to verify (6.17) for all finite sums $z=\sum_{j=1}^{r} c_{j} z_{n_{j}}$ and $y=\sum_{j=1}^{r} c_{j} y_{j}$. Put $F^{*}(x)=\sum_{s \in F} x(s)$ for $x=\sum_{s=1}^{\infty} x(s) e_{s} \in X(w)$, whenever $F \subset \mathbf{N}$ is a finite interval. Suppose that $F_{1}<F_{2}<\ldots<F_{m}$ are finite intervals for which $\sum_{i=1}^{m} w_{i}\left|F_{i}^{*}(z)\right|=\|z\|$. The non-zero terms $\left|F_{i}^{*}(z)\right|$ have the form $\left|c_{j}\right|$ or $\left|c_{j}-c_{k}\right|$ for suitable $j<k$. Indeed, there is no contribution to $F_{i}^{*}(z)$ from the terms $c_{l}\left(e_{2 n_{l}-1}-e_{2 n_{l}}\right)$, where both $2 n_{l}-1,2 n_{l} \in F_{i}$.

If $i$ is such that $\left|F_{i}^{*}(z)\right|=\left|c_{j}\right|$, then we may replace $F_{i}$ by $G_{i}=\left\{2 n_{j}-1\right\}$ or $G_{i}=\left\{2 n_{j}\right\}$ without affecting $\left|F_{i}^{*}(z)\right|=\left|c_{j}\right|$. The choice of $2 n_{j}-1$ or $2 n_{j}$ is according to which of these indices contributes the term $\left|c_{j}\right|$. Thus $G_{i}^{*}(y)=b_{j} c_{j}$ or $G_{i}^{*}(y)=a_{j} c_{j}$, so that (iii) yields

$$
\left|G_{i}^{*}(y)\right| \geq \min \left\{\left|a_{j}\right|,\left|b_{j}\right|\right\} \cdot\left|c_{j}\right| \geq \frac{8}{10}\left|c_{j}\right|=\frac{8}{10}\left|F_{i}^{*}(z)\right| .
$$

If $\left|F_{i}^{*}(z)\right|=\left|c_{j}-c_{k}\right|$ for some $j<k$, then we consider two singletons $G_{i, 1}<G_{i, 2}$ of the preceding type instead of $F_{i}$. In this case we get as above that

$$
\left|F_{i}^{*}(z)\right|=\left|c_{j}-c_{k}\right| \leq\left|c_{j}\right|+\left|c_{k}\right| \leq \frac{10}{8}\left(\left|G_{i, 1}^{*}(y)\right|+\left|G_{i, 2}^{*}(y)\right|\right) .
$$

Put $A=\left\{i:\left|F_{i}^{*}(z)\right|=\left|c_{j}\right|\right.$ for some $\left.j\right\}$ and $B=\left\{i:\left|F_{i}^{*}(z)\right|=\left|c_{j}-c_{k}\right|\right.$ for some $j<k\}$. We get from (6.18) and (6.19) that

$$
\begin{aligned}
\|z\| & =\sum_{i \in A} w_{i}\left|F_{i}^{*}(z)\right|+\sum_{i \in B} w_{i}\left|F_{i}^{*}(z)\right| \\
& \leq \frac{10}{8}\left(\sum_{i \in A} w_{i}\left|G_{i}^{*}(y)\right|+\sum_{i \in B} w_{i}\left|G_{i, 1}^{*}(y)\right|\right)+\frac{10}{8} \sum_{i \in B} w_{i}\left|G_{i, 2}^{*}(y)\right| \leq \frac{20}{8}\|y\| .
\end{aligned}
$$

Here $\sum_{i \in B} w_{i}\left|G_{i, 2}^{*}(y)\right| \leq\|y\|$, since the weight sequence $\left(w_{i}\right)$ is non-increasing.

Remark 6.15. The arguments for Examples 6.12 and 6.14 demonstrate that $d(w, 1)$ and $X(w)$ even fail to have the unbounded W.A.P.

The inner W.A.P. (see section 5) is more difficult to study. Our final example shows that the dual $J T^{*}$ of the James tree space does not have the inner W.A.P. We will require the following facts from [LS, Thm. 1]: JT has a predual $B$ and $B^{* *} / B=\ell^{2}(\Gamma)$ isometrically, where $\Gamma$ is the uncountable collection of all branches of $\mathcal{T}$. In particular, $J T^{* * *} / J T^{*}=\ell^{2}(\Gamma)$.

Example 6.16. $J T^{*}$ does not have the inner WAP.

Proof. The argument is a modification of that of [T2, 1.4] for the Johnson-Lindenstrauss space. Let $q: J T^{*}=B^{* *} \rightarrow \ell^{2}(\Gamma)$ be the weakly compact quotient map. Suppose to the contrary that there is a sequence $\left(V_{n}\right) \subset W\left(J T^{*}\right)$ such that

$$
\lim _{n \rightarrow \infty}\left\|q-q V_{n}\right\|=0 .
$$


Recall next that any weakly compact set $D \subset J T^{*}$ is norm separable, since $(D, w)$ is metrizable in this case. This is based on the fact that $J T$ is a separable space not containing any copies of $\ell^{1}$ (see [LS, Cor. 1] or [FG. 3.a.8]), so that $J T$ is $w^{*}$-sequentially dense in $J T^{* *}$ by the Main Theorem of [OR].

Deduce that the closure $\overline{q\left(V_{n} B_{J T^{*}}\right)}$ is a norm separable set in $\ell^{2}(\Gamma)$ for $n \in \mathbf{N}$. Thus (6.20) implies that $B_{\ell^{2}(\Gamma)}=\overline{q\left(B_{J T^{*}}\right)}$ is also norm separable by approximation. This contradicts the non-separability of $\ell^{2}(\Gamma)$.

If $E$ has the inner W.A.P., then $W(E)$ has a B.R.A.I. by [BD, Prop. 11.2] (cf. also the proof of Proposition 2.5(i)). Thus Example 6.16 and [LW, Cor. 2.4] suggest

Problems 6.17. (i) Does $J$ have the inner W.A.P.?

(ii) Does $J T$ have the inner W.A.P.?

(iii) Does $J T^{*}$ have the W.A.P.?

\section{ACKNOWLEDGEMENTS}

The second-named author is greatly indebted to E. Odell and H. P. Rosenthal for their hospitality during his visit to the Department of Mathematics, the University of Texas at Austin, in late 2002.

\section{REFERENCES}

[A] A. Andrew: James' quasi-reflexive space is not isomorphic to any subspace of its dual, Israel J. Math. 38 (1981), 276-282. MR0617674 (82g:46028)

[ArT] S.A. Argyros and A. Tolias: Methods in the theory of hereditarily indecomposable Banach spaces, Mem. Amer. Math. Soc. 170 (2004), no. 806, vi+114 pp. MR2053392

[AT] K. Astala and H.-O. Tylli: Seminorms related to weak compactness and to Tauberian operators, Math. Proc. Cambridge Phil. Soc. 107 (1990), 367-375. MR 1027789(91b:47016)

[AH] P. Azimi and J.N. Hagler: Examples of hereditarily $\ell^{1}$ Banach spaces failing the Schur property, Pacific J. Math. 122 (1986), 287-297. MR0831114 (87f:46030)

[BHO] S.F. Bellenot, R. Haydon and E. Odell: Quasi-reflexive and tree spaces constructed in the spirit of R.C. James, Contemp. Math. 85 (1989), 19-43. MR.0983379 (89m:46014)

[BD] F.F. Bonsall and J. Duncan: Complete normed algebras. Ergebnisse der Mathematik vol. 80 (Springer, 1973). MR 0423029 (54:11013)

[B] J. Bourgain: New classes of $\mathcal{L}^{p}$-spaces. Lecture Notes in Mathematics vol. 889 (SpringerVerlag, 1981). MR0639014 (83j:46028)

[CLL] P.G. Casazza, B.L. Lin and R.H. Lohman: On James' quasi-reflexive Banach space, Proc. Amer. Math. Soc. 67 (1977), 265-271. MR0458129(56:16332)

[D] H.G. Dales: Banach algebras and automatic continuity. London Mathematical Society Monographs vol. 24 (Oxford University Press, 2000). MR1816726 (2002e:46001)

[Di] J. Diestel: A survey of results related to the Dunford-Pettis property, Contemp. Math. 2 (1980), 15-60. MR.0621850 (82i:46023)

[FG] H. Fetter and B. Gamboa de Buen: The James Forest, London Math. Soc. Lecture Notes 236 (Cambridge University Press, 1997). MF 1474498 (98k:46013)

[GST] M. Gonzalez, E. Saksman and H.-O. Tylli: Representing non-weakly compact operators, Studia Math. 113 (1995), 265-282. MR1330211 (96i:47078)

[GW] N. Grønbæk and G.A. Willis: Approximate identities in Banach algebras of compact operators. Canad. Math. Bull. 36 (1993), 45-53. MR1205894 (94b:46076)

[J1] R.C. James: A non-reflexive Banach space isometric with its second conjugate space. Proc. Nat. Acad. Sci. U.S.A. 37 (1951), 174-177. MR0044024 (13:356d)

[J2] R.C. James: A separable somewhat reflexive Banach space with nonseparable dual, Bull. Amer. Math. Soc. 80 (1974), 738-743. MR0417763(54:5811)

[L] I.E. Leonard: Banach sequence spaces, J. Math. Anal. Appl. 54 (1976), 245-265. MR0420216 (54:8230) 
[LNO] A. Lima, O. Nygaard and E. Oja: Isometric factorization of weakly compact operators and the approximation property, Israel J. Math. 119 (2002), 325-348. MR1802659 (2002b:46031)

[LS] J. Lindenstrauss and C. Stegall: Examples of separable spaces which do not contain $\ell^{1}$ and whose duals are not separable, Studia Math. 54 (1975), 81-105. MR 0390720 (52:11543)

[LT] J. Lindenstrauss and L. Tzafriri: Classical Banach spaces I. Sequence spaces. Ergebnisse der Mathematik vol. 92 (Springer, 1977). MR0500056 (58:17766)

[LW] R.J. Loy and G.A. Willis: Continuity of derivations on $B(E)$ for certain Banach spaces $E$, J. London Math. Soc. 40 (1989), 327-346. MR1044280 (91f:46069)

[OR] E. Odell and H.P. Rosenthal: A double dual characterization of separable Banach spaces containing $\ell^{1}$, Israel J. Math. 20 (1975), 375-384. MF0377482(51:13654)

[Ph] R.R. Phelps: Lectures on Choquet's Theorem (2nd edition). Lecture Notes in Mathematics vol. 1757 (Springer, 2001). MR 1835574 (2002k:46001)

[P] G. Pisier: The dual $J^{*}$ of the James space has cotype 2 and the Gordon Lewis property, Math. Proc. Cambridge Phil. Soc. 103 (1988), 323-331. MR0923685 (89a:46041)

[PQ] G. Pisier and Q. Xu: Random series in the interpolation spaces between the spaces $v_{p}$, in Geometric Aspects of Functional Analysis 1985-86 (J. Lindenstrauss and V. Milman, eds.) Lecture Notes in Mathematics vol. 1267 (Springer, 1987), pp. 185-209. MR0907695 (89d:46011)

[Pt] V. Ptak: A combinatorial theorem on systems of inequalities and its applications to analysis, Czech. Math. J. 84 (1959), 629-630. MR0110007 (22:890)

[R] O. Reinov: How bad can a Banach space with the approximation property be? Math. Notes 33 (1983), 427-434. MR0709222 (85m:46018)

[T1] H.-O. Tylli: The essential norm of an operator is not self-dual, Israel J. Math. 91 (1995), 93-110. MR:1348307|(96f:47017)

[T2] H.-O. Tylli: Duality of the weak essential norm, Proc. Amer. Math. Soc. 129 (2001), 1437-1443. MR1814170 (2002c:47021)

[W] M. Wojtowicz: On the James space $J(X)$ for a Banach space $X$, Comment. Math. Prace Mat. 23 (1983), 183-188. MR0709187 (84h:46017)

Department of Mathematics, The University of Texas at Austin, Austin, Texas 78712

E-mail address: odell@math.utexas.edu

Department of Mathematics and Statistics, University of Helsinki, P.B. 68 (Gustaf HÄLlSTRÖMIN KATU 2B), FIN-00014 FinLAND

E-mail address: hojtylli@cc.helsinki.fi 